\title{
Minimal $f$-divergence martingale measures and optimal portfolios for exponential Levy models with a change-point
}

\author{
October 4, 2018 \\ S. Cawston! and L. Vostrikova ${ }^{2}$
}

\begin{abstract}
We study exponential Levy models with change-point which is a random variable, independent from initial Levy processes. On canonical space with initially enlarged filtration we describe all equivalent martingale measures for changepoint model and we give the conditions for the existence of f-minimal equivalent martingale measure. Using the connection between utility maximisation and $f$-divergence minimisation, we obtain a general formula for optimal strategy in change-point case for initially enlarged filtration and also for progressively enlarged filtration when the utility is exponential. We illustrate our results considering the Black-Scholes model with change-point.
\end{abstract}

KEY WORDS AND PHRASEs: f-divergence, exponential Levy models, changepoint, optimal portfolio

MSC 2010 subject classifications: 60G46, 60G48, 60G51, 91B70

\section{Introduction}

The parameters of financial models are generally highly dependent on time : a number of events (for example the release of information in the press, changes in the price of raw materials or the first time a stock price hits some psychological level) can trigger a change in the behaviour of stock prices. This time-dependency of the parameters can often be described using a piece-wise constant function : we will call this case a change-point model. In this context, an important problem in financial mathematics will be option pricing and hedging. Of course, the time of change (change-point) for

\footnotetext{
1,2 LAREMA, Département de Mathématiques, Université d'Angers, 2, Bd Lavoisier - 49045, Angers Cedex 01.

${ }^{1}$ E-mail: suzanne.cawston@univ-angers.fr ${ }^{2}$ E-mail: lioudmila.vostrikova@univ-angers.fr
} 
the parameters is not explicitly known, but it is often possible to make reasonable assumptions about its nature and use statistical tests for its detection.

Change-point problems have a long history, probably beginning with the papers of Page [44, 45] in an a-posteriori setting, and of Shiryaev [53] in a quickest detection setting. The problem was later considered in many papers, see for instance [14], 48], [21], [47], 3], [58], [41] and also the book [2] and references there. In the context of financial mathematics, the question was investigated in [30], [8], [29], [22], [15], [56], [57, [37], [59] and was often related to a quickest detection approach.

It should be noticed that not only quickest detection approach is interesting in financial mathematics, and this fact is related with pricing and hedging of so called default models (see [1], [17] and references there). In mentioned papers a number of very important results was obtained but for the processes without jump part or with only one jump.

The models with jumps, like exponential Levy models, in general, compromise the uniqueness of an equivalent martingale measure when such measure exists. So, one has to choose in some a way an equivalent martingale measure to price. Many approaches have been developed and various criteria suggested for this choice of martingale measure, for example risk-minimization in an $L^{2}$-sense [39] [19], [51], [52, Hellinger integrals minimization [11], [12], 28], entropy minimization [42], 20], [18], $f^{q}$-martingale measures [33] or Esscher measures [31].

All these approaches can be considered in unified way using so called $f$-divergences, introduced by Ciszar [13] and investigated in a number of papers and books (see for instance [40] and references there). We recall that for $f$ a convex function on $\mathbb{R}^{+, *}$ and two measures $Q$ and $P$ such that $Q<<P$, the $f$-divergence of $Q$ with respect to $P$ is defined as

$$
f(Q \mid P)=\mathbb{E}_{P}\left[f\left(\frac{d Q}{d P}\right)\right]
$$

where $\frac{d Q}{d P}$ is Radon-Nikodym density of $Q$ with respect to $P$, and $E_{P}$ is the expectation with respect to $P$. We recall that the utility maximisation is closely related to $f$-divergence minimisation via Fenchel-Legendre transform and this will be one of essential points to obtain an optimal strategy.

The aim of this paper is to study $f$-divergence minimal martingale measures and optimal portfolios from the point of view of utility maximization, for exponential Levy model with change-point where the parameters of the model before and after the change are known and a change-point itself is a random variable, independent from initial Levy processes.

We start by describing our model in more detail. We assume the financial market consists of a non-risky asset $B$ with interest rate $\left(r_{t}\right)_{t \geq 0}$, namely

$$
B_{t}=B_{0} \exp \left(\int_{0}^{t} r_{s} d s\right)
$$

where

$$
r_{t}=r \mathbf{1}_{\{\tau>t\}}+\tilde{r} \mathbf{1}_{\{\tau \leq t\}},
$$


with $r, \tilde{r}$ interest rates before and after change-point $\tau$, and one-dimensional risky asset $S=\left(S_{t}\right)_{t \geq 0}$

$$
S_{t}=S_{0} \exp \left(X_{t}\right)
$$

where $X$ is a stochastic process obtained by pasting in $\tau$ of two Levy processes $L$ and $\tilde{L}$ together:

$$
X_{t}=L_{t} \mathbf{1}_{\{\tau>t\}}+\left(L_{\tau}+\tilde{L}_{t}-\tilde{L}_{\tau}\right) \mathbf{1}_{\{\tau \leq t\}}
$$

Here and further $L$ and $\tilde{L}$ supposed to be independent Levy processes with characteristics $(b, c, \nu)$ and $(\tilde{b}, \tilde{c}, \tilde{\nu})$ respectively which are independent from $\tau$ ( for more details see [50]). To avoid unnecessary complications we assume up to now that for change-point model $r$ and $\tilde{r}$ in (2) are equal to zero, and that $S_{0}=1$.

To describe a probability space on which the process $X$ is well-defined, we consider $(D, \mathcal{G}, \mathbb{G})$ the canonical space of right-continuous functions with left-hand limits equipped with its natural filtration $\mathbb{G}=\left(\mathcal{G}_{t}\right)_{t \geq 0}$ which satisfies standard conditions: it is right-continuous, $\mathcal{G}_{0}=\{\emptyset, D\}, \bigvee_{t \geq 0} \mathcal{G}_{t}=\mathcal{G}$. On the product of such canonical spaces we define two independent Levy processes $L=\left(L_{t}\right)_{t \geq 0}$ and $\tilde{L}=\left(\tilde{L}_{t}\right)_{t \geq 0}$ with characteristics $(b, c, \nu)$ and $(\tilde{b}, \tilde{c}, \tilde{\nu})$ respectively and denote by $P$ and $\tilde{P}$ their respective laws which are assumed to be locally equivalent: $P \stackrel{\text { loc }}{\sim} \tilde{P}$. As we will consider the market on a fixed finite time interval, we are really only interested in $\left.P\right|_{\mathcal{G}_{T}}$ and $\left.\tilde{P}\right|_{\mathcal{G}_{T}}$ for a fixed $T \geq 0$ and the distinction between equivalence and local equivalence does not need to be made.

Our change-point will be represented by an independent random variable $\tau$ of law $\alpha$ taking values in $([0, T], \mathcal{B}([0, T])$. The set $\{\tau=T\}$ corresponds to the situation when the change-point does not take place, or at least not on the interval we are studying.

On the probability space $(D \times D \times[0, T], \mathcal{G} \times \mathcal{G} \times \mathcal{B}([0, T], P \times \tilde{P} \times \alpha)$ we define a measurable map $X$ by (44) and we denote by $\mathbb{P}$ its law. In what follows we use $\mathbb{E}$ mainly for the expectation with respect to $\mathbb{P}$ but this notation will be also used for the expectation with respect to $P \times \tilde{P} \times \alpha$.

From point of view of observable processes we can have the following situations. If we observe only the process $X$ then the natural probability space to work is $(D, \mathcal{G}, \mathbb{P})$ equipped with the right-continuous version of the natural filtration $\mathbb{G}=\left(\mathcal{G}_{t}\right)_{t \geq 0}$ where $\mathcal{G}_{t}=\sigma\left\{X_{s}, s \leq t\right\}$ for $t \geq 0$. Now, if we observe not only the process $X$ but also some complementary variables related with $\tau$ then we can take it in account by the enlargement of the filtration. First we consider the filtration $\mathbb{H}$ given by $\mathcal{H}_{t}=\sigma\left(\mathbf{1}_{\{\tau \leq s\}}, s \leq t\right)$ and note that $\mathcal{H}_{T}=\sigma(\tau)$. Then we introduce two filtrations: the initially enlarged filtration $\mathbb{F}=\left(\mathcal{F}_{t}\right)_{t \geq 0}$

$$
\mathcal{F}_{0}=\mathcal{G}_{0} \vee \mathcal{H}_{T}, \quad \mathcal{F}_{t}=\bigcap_{s>t}\left(\mathcal{G}_{s} \vee \mathcal{H}_{T}\right)
$$

and the progressively enlarged filtration $\hat{\mathbb{F}}=\left(\hat{\mathcal{F}}_{t}\right)_{t \geq 0}$ which satisfies :

$$
\hat{\mathcal{F}}_{0}=\mathcal{G}_{0} \vee \mathcal{H}_{0}, \quad \hat{\mathcal{F}}_{t}=\bigcap_{s>t}\left(\mathcal{G}_{s} \vee \mathcal{H}_{s}\right)
$$

In the case of additional information the most natural filtration from the point of view of observable events would be $\hat{\mathbb{F}}$. However, we will see that it is much easier to start by 
working with the initially enlarged filtration and then come back to the progressively enlarged filtration.

The paper is organized in the following way. We start by recalling some known facts about f-divergence minimal martingale measures. Then, on mentioned probability space and for initially enlarged filtration we describe all equivalent martingale measures. We introduce as hypotheses, such properties of $f$-divergence minimal martingale measures as a preservation of Levy property and a scaling property. As known, these properties are verified by all Levy processes and common $f$-divergences such that $f^{\prime \prime}(x)=a x^{\gamma}, a>0, \gamma \in \mathbb{R}$. We recall that these functions are those for which there exists $A>0$ and real $B, C$ such that $f(x)=A f_{\gamma}(x)+B x+C$ where

$$
f_{\gamma}(x)=\left\{\begin{array}{l}
c_{\gamma} x^{\gamma+2} \text { if } \gamma \neq-1,-2 \\
x \ln (x) \text { if } \gamma=-1 \\
-\ln (x) \text { if } \gamma=-2
\end{array}\right.
$$

and $c_{\gamma}=\operatorname{sign}[(\gamma+1) /(\gamma+2)]$. The question of preservation of Levy property was considered in details in [5] and it was shown that the class of $f$-divergences preserving Levy property is larger then common $f$-divergences. Then, the conditions for existence and the expression of Radon-Nikodym density $Z_{T}^{*}$ of $f$-minimal martingale measure for change-point model is given in Theorem 1, The result is applied to Black-Scholes change-point model which became an incomplete model.

In 3. we present some known facts about utility maximisation. Then we give a decomposition formula for $f^{\prime}\left(Z_{T}^{*}\right)$ which allow us via the result of [27] to identify optimal strategy (see Theorem 3). We illustrate this result by considering again the Black-Scholes model with a change-point.

\section{$2 \quad f$-minimal MME's for change-point model}

We start by recalling some known facts about $f$-divergence minimal martingale measures. Then, we describe all locally equivalent martingale measures (EMMs) leaving on our probability space equipped with initially enlarged filtration for change-point model, and in particular in relation to the sets of EMMs for the two associated Levy models $L$ and $\tilde{L}$ which we denote by $\mathcal{M}(P)$ and $\mathcal{M}(\tilde{P})$ respectively.

\subsection{EMMs for exponential Levy models}

The aim of this part is to show how one can find Girsanov parameters of $f$-divergence minimal martingale measure. As we will see later, to write down Radon-Nikodym density $Z_{T}^{*}$ of $f$-minimal MME for change-point model we need only to know the Girsanov parameters of $f$-minimal MME for associated Levy models $L$ and $\tilde{L}$, the parameters of mentioned Levy processes $L$ and $\tilde{L}$ and the law of $\tau$.

Let now $L=\left(L_{t}\right)_{t \geq 0}$ be Levy process with parameters $(b, c, \nu)$ where $b$ is the drift parameter, $c$ is the diffusion parameter and $\nu$ is the Levy measure, i.e. the measure on 
$\mathbb{R} \backslash\{0\}$ which satisfies

$$
\int_{\mathbb{R}}\left(x^{2} \wedge 1\right) \nu(d x)<+\infty .
$$

We recall that the characteristic function of $L_{t}$ for $t \in \mathbb{R}^{+}$and $u \in \mathbb{R}$ is given then by:

$$
\phi_{t}(u)=\mathbb{E} e^{i u L_{t}}=e^{\psi(u) t}
$$

and in turn, the characteristic exponent

$$
\psi(u)=i u b-\frac{1}{2} c u^{2}+\int_{\mathbb{R}}(\exp (i u x)-1-i u l(x)) \nu(d x)
$$

where from now on, $l$ is the truncation function.

\subsection{Esscher measures}

Esscher measures play very important role in actuarial theory as well as in the option pricing theory and they were studied in [36], [42], [20]. Let

$$
D=\left\{u \in \mathbb{R} \mid E_{P} e^{u L_{1}}<\infty\right\}
$$

where $E_{P}$ is the expectation with respect to the physical measure $P$. Then for $u \in D$ we define Esscher measure $P^{E S}$ of the parameter $u$ and risk process $\left(L_{t}\right)_{t \geq 0}$ by : for $t \geq 0$

$$
\frac{d P_{t}^{E S}}{d P_{t}}=\frac{e^{u L_{t}}}{E_{P}\left[e^{u L_{t}}\right]}
$$

It is known that $\left(e^{-r t} S_{t}\right)_{t \geq 0}$ is a martingale under $Q=P^{E S}$ iff

$$
b+\left(\frac{1}{2}+u\right) c+\int_{\mathbb{R}^{*}}\left(\left(e^{x}-1\right) e^{u x}-l(x)\right) \nu(d x)=r
$$

We can easily see that the left-hand side of (9) is continuous increasing function of $u$ on $D$ and that $D$ is an interval. If we denote by $g$ the left-hand side of (9) and by $\bar{d}$ right end point of $D$, then the conditions

$$
\lim _{u \rightarrow \bar{d}} g(u)>r, \lim _{u \rightarrow-\infty} g(u)<r
$$

insure the existence of solution of (91). If, in addition, $g$ is strictly increasing, the solution will be unique. More about existence and uniqueness of solution of (9) see 31] and [36].

Suppose that the solution of (9) exists and is denoted by $\beta^{*}$. Then Esscher martingale measure is $Q^{*}=P^{E S}\left(\beta^{*}\right)$ and we show that the Girsanov parameters of $Q^{*}$ are: $\beta^{Q}=\beta^{*} Y^{Q}=e^{\beta^{*} x}$. In fact,

$$
\zeta_{t}=\frac{d Q_{t}^{*}}{d P_{t}}=\frac{e^{\beta^{*} L_{t}}}{\phi\left(-i \beta^{*} t\right)}
$$


From the formula (10) we see that

$$
\frac{\zeta_{t}}{\zeta_{t-}}=e^{\beta^{*} \Delta L_{t}}
$$

and according to Girsanov theorem

$$
Y^{Q^{*}}=M_{\mu}^{P}\left(e^{\beta^{*} \Delta L} \mid \tilde{\mathcal{P}}\right)=e^{\beta^{*} x} .
$$

We use Ito formula to find $\zeta^{c}$ :

$$
\zeta_{t}^{c}=\int_{0}^{t} \frac{\beta^{*} \exp \left(\beta^{*} L_{s-}\right)}{\phi\left(-i \beta^{*} s\right)} d L_{s}^{c}
$$

and, hence,

$$
\beta_{t}^{Q^{*}}=\frac{1}{\zeta_{t-}} \frac{d\left\langle\zeta^{c}, L^{c}\right\rangle_{t}}{d C_{t}}=\beta^{*}
$$

\subsection{Minimal entropy measures}

We recall that for two equivalent probability measures $Q$ and $P$, the relative entropy of $Q$ with respect to $P$ ( or Kullback-Leibler information in $Q$ with respect to $P$ ) is:

$$
H(Q \mid P)=E_{Q}\left(\ln \left(\frac{d Q}{d P}\right)\right)=E_{P}\left(\frac{d Q}{d P} \ln \left(\frac{d Q}{d P}\right)\right)
$$

and it is $f$-divergence with $f(x)=x \ln (x)$. We are interested in minimal entropy martingale measure, i.e. the measure $P^{M E}$ such that $\left(e^{-r t} S_{t}\right)_{t \geq 0}$ is a $P^{M E}$-martingale, and that for all $Q$ martingale measures

$$
H\left(P^{M E} \mid P\right) \leq H(Q \mid P)
$$

It turns out (cf.[20]) that in the case of Levy processes $P^{M E}$ is nothing else as Esscher measure but for another risk process $\left(\hat{L}_{t}\right)_{t \geq 0}$, namely for the process appearing in the representation:

$$
S_{t}=S_{0} \mathcal{E}(\hat{L})_{t}
$$

where $\mathcal{E}(\cdot)$ is Dolean's-Dade exponential,

$$
\mathcal{E}(\hat{L})_{t}=\exp \left(\hat{L}_{t}-\frac{1}{2}\langle\hat{L}\rangle_{t}\right) \prod_{0 \leq s \leq t}\left(1+\Delta \hat{L}_{s}\right) e^{-\Delta \hat{L}_{s}}
$$

Now let $D=\left\{u \in \mathbb{R} \mid E_{P} e^{u \hat{L}_{1}}<\infty\right\}$ and let us introduce Esscher measure corresponding to the risk process $\hat{L}$ and $u \in D$ : for $t \geq 0$

$$
\frac{d P_{t}^{M E}}{d P_{t}}=\frac{e^{u \hat{L}_{t}}}{E_{P}\left[e^{u \hat{L}_{t}}\right]}
$$


We can write down the characteristics of $\hat{L}$ to show that $\hat{L}$ is again Levy process. Then, $\left(e^{-r t} S_{t}\right)_{t \geq 0}$ is a martingale under $P^{M E}$ iff

$$
b+\left(\frac{1}{2}+u\right) c+\int_{\mathbb{R}}\left(\left(e^{x}-1\right) e^{u\left(e^{x}-1\right)}-l(x)\right) d \nu=r
$$

We can easily see that the left-hand side of (11) is continuous increasing function of $u$ on $D$ and that $D$ is an interval. If we denote by $g$ the left-hand side of (11) and $\bar{d}$ is right end point of $D$, then the conditions

$$
\lim _{u \rightarrow \bar{d}} g(u)>r, \lim _{u \rightarrow-\infty} g(u)<r
$$

insure the existence of solution of (11). In addition, if $g$ is strictly increasing, the solution is unique. More about existence and uniqueness of solution of (11) see [31] and [36].

Suppose that $\beta^{*}$ is a unique solution of (11), then the Girsanov parameters of a minimal entropy martingale measure $Q^{*}$ are: $\left(\beta^{*}, Y^{*}\right)$ where $Y^{*}(x)=e^{\beta^{*}\left(e^{x}-1\right)}$ for $x \in \mathbb{R}^{*}$.

\section{$2.4 f_{q}$ - martingale measures}

Let $Q$ and $P$ be two probability measures, $Q<<P$ and let $f_{q}$ be $f$-divergence defined by

$$
f_{q}(x)= \begin{cases}-x^{q}, & \text { if } \quad 0<q<1 \\ x^{q}, & \text { if } \quad q<0 \text { or } q>1\end{cases}
$$

It is not difficult to see that such $f$ is a strictly convex function. It was shown in [11], [12], 33] that when $P$ is the law of a Levy process, the Girsanov parameters $\left(\beta_{q}^{*}, Y_{q}^{*}\right)$ of the measure $Q_{q}^{*}$ minimising $f_{q}$-divergence are independent on $(t, \omega)$.

It can be also shown that if $L$ is not monotone Levy process and if we allow as $Q_{q}^{*}$ not only equivalent, but also absolute continuous measures, then the Girsanov parameters $\left(\beta_{q}^{*}, Y_{q}^{*}\right)$ are unique minimizers of the function

$$
k(\beta, Y)=\frac{q(q-1)}{2} \beta^{2} c+\int_{\mathbb{R}}\left(Y^{q}(x)-1-q(Y(x)-1)\right) \nu(d x)
$$

under the constraint

$$
b+c \beta+\int_{\mathbb{R}}(x Y(x)-l(x)) \nu(d x)=0
$$

on the set

$$
\mathcal{A}=\left\{(\beta, Y)\left|\beta \in \mathbb{R}, Y \geq 0, \int_{\mathbb{R}}\right| x Y(x)-l(x) \mid \nu(d x)<\infty\right\}
$$

Via an application of the Kuhn-Tucker theorem it can be shown that

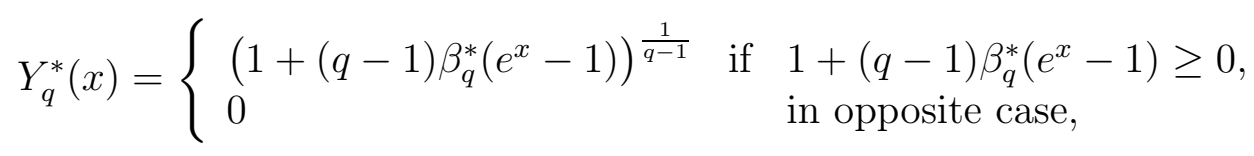


where $\beta_{q}^{*}$ is the first Girsanov parameter which can be find from the constraint. We remark that if in addition

$$
\operatorname{supp}(\nu) \subseteq\left\{x: 1+(q-1) \beta_{q}^{*}\left(e^{x}-1\right)>0\right\}
$$

then $Q_{q}^{*}$ is equivalent to $P$.

\subsection{EMMs for change-point model}

We assume that the sets $\mathcal{M}(P)$ and $\mathcal{M}(\tilde{P})$ are non-empty. Let $Q \in \mathcal{M}(P)$ and $\tilde{Q} \in \mathcal{M}(\tilde{P})$. We introduce the Radon-Nikodym density processes $\zeta=\left(\zeta_{t}\right)_{t \geq 0}$ and $\tilde{\zeta}=\left(\tilde{\zeta}_{t}\right)_{t \geq 0}$ given by

$$
\zeta_{t}=\frac{d Q_{t}}{d P_{t}}, \quad \tilde{\zeta}_{t}=\frac{d \tilde{Q}_{t}}{d \tilde{P}_{t}}
$$

where $Q_{t}, P_{t}, \tilde{Q}_{t}, \tilde{P}_{t}$ stand for the restrictions of the corresponding measures to the $\sigma$ algebra $\mathcal{F}_{t}$.

We also introduce for all $t>0$

$$
y_{t}=\frac{d \tilde{P}_{t}}{d P_{t}}
$$

then

$$
Y_{t}=\mathbf{1}_{\llbracket 0, \tau \rrbracket}(t)+\frac{y_{t}}{y_{\tau}} \mathbf{1}_{\rrbracket \tau,+\infty \llbracket}(t)
$$

We remark that the measure $\mathbb{P}$ which is the law of $X$ verify for $t \geq 0$ :

$$
\frac{d \mathbb{P}_{t}}{d P_{t}}=Y_{t}
$$

To describe all EMMs leaving on our space we define the process $z=\left(z_{t}\right)_{t \geq 0}$ given by

$$
z_{t}=\zeta_{t} \mathbf{1}_{\llbracket 0, \tau \rrbracket}(t)+\zeta_{\tau} \frac{\tilde{\zeta}_{t}}{\tilde{\zeta}_{\tau}} \mathbf{1}_{\rrbracket \tau,+\infty \llbracket}(t)
$$

Finally, we consider the measure $\mathbb{Q}$ such that

$$
\frac{d \mathbb{Q}_{t}}{d \mathbb{P}_{t}}=c(\tau) z_{t}
$$

where $c(\cdot)$ is a measurable function $[0, T] \rightarrow \mathbb{R}^{+, *}$ with $\mathbb{E} c(\tau)=1$.

Proposition 1. A measure $\mathbb{Q}$ is an equivalent martingale measure for the exponential model (4) related to the process $X$ iff its density process has the form (14).

Proof First we show that the process $Z=\left(Z_{t}\right)_{t \geq 0}$ given by

$$
Z_{t}=c(\tau) z_{t}
$$

is a density process with respect to $\mathbb{P}$ and that the process $S=\left(S_{t}\right)_{t \geq 0}$ such that

$$
S_{t}=e^{L_{t}} \mathbf{1}_{\llbracket 0, \tau \rrbracket}(t)+S_{\tau} e^{\tilde{L}_{t}-\tilde{L}_{\tau}} \mathbf{1}_{\rrbracket \tau,+\infty \llbracket}(t)
$$


is a $(\mathbb{Q}, \mathbb{F})$ - martingale.

We begin by noticing that if $M, \tilde{M}$ are two strictly positive martingales on the same filtered probability space and $\tau$ is a stopping time independent of $M$ and $\tilde{M}$, then $N=\left(N_{t}\right)_{t \geq 0}$ such that

$$
N_{t}=c(\tau)\left[M_{t} \mathbf{1}_{\llbracket 0, \tau \rrbracket}(t)+M_{\tau} \frac{\tilde{M}_{t}}{\tilde{M}_{\tau}} \mathbf{1}_{\rrbracket \tau,+\infty \llbracket}(t)\right]
$$

is again a martingale. This fact, for example, can be proved by the conditioning with respect to $\mathcal{H}_{T}$ and use the facts that $M$ and $\tilde{M}$ are martingales. To show that $Z$ is a $(\mathbb{P}, \mathbb{F})$-martingale, we prove an equivalent fact that $\left(Y_{t} Z_{t}\right)_{t \geq 0}$ is a $(\underset{\tau}{P}, \mathbb{F})$ - martingale. But this follows from the previous remark taking $M_{t}=\zeta_{t}$ and $\tilde{M}_{t}=\tilde{\zeta}_{t} y_{t}$ and using (12), (13), (15). Furthermore, taking conditional expectation with respect to $\mathcal{H}_{T}$ and using the fact that $\zeta$ and $\tilde{\zeta}$ are density processes independent from $\tau$, we see that $\mathbb{E} Z_{t}=1$. To show that $S=\left(S_{t}\right)_{t \geq 0}$ is $(\mathbb{Q}, \mathbb{F})$-martingale we establish that $\left(Y_{t} Z_{t} S_{t}\right)_{t \geq 0}$ is a $(P, \mathbb{F})$ - martingale. For this we use the same remark with $M_{t}=e^{L_{t}} \zeta_{t}$ and $\tilde{M}_{t}=y_{t} \tilde{\zeta}_{t} e^{\tilde{L}_{t}}$.

Conversely, $Z$ is the density of any equivalent martingale measure if and only if $\left(Z_{t} S_{t}\right)_{t \geq 0}$ is a $(\mathbb{P}, \mathbb{F})$ - martingale. But the last fact is equivalent to the fact that for any bounded stopping time $\sigma$,

$$
\mathbb{E}\left(Z_{\sigma} S_{\sigma}\right)=1
$$

Replacing $\sigma$ by $\sigma \wedge \tau$ in previous expression we deduce that $\left(Z_{t \wedge \tau}\right)_{t \geq 0}$ is the density of a martingale measure for $\left(e^{L_{t \wedge \tau}}\right)_{t \geq 0}$. In the same way, using the martingale properties of $Z$ we get for any bounded stopping time $\sigma$ that

$$
\mathbb{E}\left(\frac{Z_{\sigma} S_{\sigma}}{Z_{\sigma \wedge \tau} S_{\sigma \wedge \tau}}\right)=1
$$

and so $\left(\frac{Z_{t}}{Z_{t \wedge \tau}}\right)_{t \geq \tau}$ is the density of an equivalent martingale measure for $\left(e^{\tilde{L}_{t}-\tilde{L}_{t \wedge \tau}}\right)_{t \geq \tau}$.

\subsection{From equivalent to $f$-divergence minimal martingale mea- sure.}

We now turn to the problem of finding $f$-divergence minimal martingale measure on time interval $[0, T]$. We recall that for a convex function $f$ on $\mathbb{R}^{+, *}$, the $f$-divergence of the restriction $Q_{T}$ of the measure $Q$ with respect to the restriction $P_{T}$ of the measure $P$ is:

$$
f\left(Q_{T} \mid P_{T}\right)=E_{P}\left[f\left(\frac{d Q_{T}}{d P_{T}}\right)\right]
$$

if the last integral exists and by convention we set it equal to $+\infty$ otherwise. We recall that $Q_{T}^{*}$ is an $f$-divergence minimal equivalent martingale measure if $f\left(Q_{T}^{*} \mid P_{T}\right)<+\infty$ and

$$
f\left(Q_{T}^{*} \mid P_{T}\right)=\inf _{Q \in \mathcal{M}(P)} f\left(Q_{T} \mid P_{T}\right)
$$

where $\mathcal{M}(P)$ is the set of locally equivalent martingale measures supposed to be nonempty. We also recall that an $f$-divergence minimal equivalent martingale measure $Q^{*}$ 
is invariant under scaling if for all $x \in \mathbb{R}^{+, *}$

$$
f\left(x Q_{T}^{*} \mid P_{T}\right)=\inf _{Q \in \mathcal{M}(P)} f\left(x Q_{T} \mid P_{T}\right)
$$

For a given exponential Levy model $S=S_{0} e^{L}$, we say that an $f$-divergence minimal martingale measure $Q^{*}$ preserves the Levy property if $L$ remains a Levy process under $Q^{*}$.

In the following theorem we give an expression for the density of the $f$-divergence minimal martingale measures in our change-point framework. We introduce the following hypotheses :

$\left(\mathcal{H}_{1}\right)$ : The $f$-divergence minimal equivalent martingale measures $Q^{*}$ and $\tilde{Q}^{*}$ relative to $L$ and $\tilde{L}$ exist.

$\left(\mathcal{H}_{2}\right)$ : The $f$-divergence minimal equivalent martingale measures $Q^{*}$ and $\tilde{Q}^{*}$ preserve the Levy property and are invariant under scaling.

$\left(\mathcal{H}_{3}\right)$ : For all $c>0$ and $t \in[0, T]$, we have $E_{Q}\left|f^{\prime}\left(c \zeta_{t}^{*}\right)\right|<\infty, E_{\tilde{Q}}\left|f^{\prime}\left(c \tilde{\zeta}_{t}^{*}\right)\right|<\infty$ where $\zeta^{*}$ and $\tilde{\zeta}^{*}$ are the densities of the $f$-minimal equivalent martingale measures $Q^{*}$ and $\tilde{Q}^{*}$ with respect to $P$ and $\tilde{P}$ respectively.

We set for $t \in[0, T]$

$$
z_{T}^{*}(t)=\zeta_{t}^{*} \frac{\tilde{\zeta}_{T}^{*}}{\tilde{\zeta}_{t}^{*}}
$$

Theorem 1. Assume that $f$ is a strictly convex function, $f \in C^{1}\left(\mathbb{R}^{+, *}\right)$, and that $\left(\mathcal{H}_{1}\right),\left(\mathcal{H}_{2}\right),\left(\mathcal{H}_{3}\right)$ hold. If the $f$ - minimal equivalent martingale measure $\mathbb{Q}^{*}$ for the change-point model (4) exists, then

$$
\frac{d \mathbb{Q}_{T}^{*}}{d \mathbb{P}_{T}}=c(\tau) z_{T}^{*}(\tau)
$$

where $c(\cdot)$ is a measurable function $[0, T] \rightarrow \mathbb{R}^{+}$such that $\mathbb{E} c(\tau)=1$.

For $c>0$, let

$$
\lambda_{t}(c)=\mathbb{E}\left[f^{\prime}\left(c z_{T}^{*}(t)\right) z_{T}^{*}(t)\right]
$$

where the expectation is taken with respect to $\mathbb{P}$ and let $c_{t}(\lambda)$ be its right-continuous inverse.

If in addition there exists $\lambda^{*}$ such that

$$
\int_{0}^{T} c_{t}\left(\lambda^{*}\right) d \alpha(t)=1
$$

then the $f$-minimal equivalent martingale measure for a change-point model exists and is given by (17) with $c^{*}(t)=c_{t}\left(\lambda^{*}\right)$ for $t \in[0, T]$. 
In particular, if $f^{\prime}(x)=a x^{\gamma}$, for $a>0$ and $\gamma \in \mathbb{R}^{+, *}$, then

$$
c^{*}(t)=\frac{\left[\mathbb{E}\left(z_{T}^{*}(t)^{\gamma+1}\right)\right]^{-\frac{1}{\gamma}}}{\int_{0}^{T}\left[\mathbb{E}\left(z_{T}^{*}(t)^{\gamma+1}\right)\right]^{-\frac{1}{\gamma}} d \alpha(t)}
$$

and for $f^{\prime}(x)=\ln (x)+1$,

$$
c^{*}(t)=\frac{e^{-\mathbb{E}\left(z_{T}^{*}(t) \ln z_{T}^{*}(t)\right)}}{\int_{0}^{T} e^{-\mathbb{E}\left(z_{T}^{*}(t) \ln z_{T}^{*}(t)\right)} d \alpha(t)} .
$$

Remark 1. Let us comment mentioned above hypotheses. Of course, the $f$-divergence minimal martingale measure not always exist. The necessary and sufficient conditions for existence of such measures for common $f$-divergences was mentioned in [5]. As it was shown in [19], [18], [11], [33], the properties mentioned in hypotheses $\left(\mathcal{H}_{2}\right)$ are satisfied for the most common $f$-divergence functions and any Levy processes. But the class of functions satisfying $\left(\mathcal{H}_{2}\right)$ is larger then just common $f$-divergences ( cf. [5]). The hypotheses $\left(\mathcal{H}_{3}\right)$ contains a number of integrability conditions needed to use scaling property. If for each $c>0$ there exist $A, B, C$ real constants such that

$$
\left|x f^{\prime}(c x)\right| \leq A|f(x)|+B x+C
$$

then $\left(\mathcal{H}_{3}\right)$ will follow automatically from the integrability condition for existence of $f$-divergence minimal equivalent martingale measure.

Remark 2. We can express the factor $c^{*}(t)$ in terms of Radon-Nikodym densities of $f$-divergence measures of the processes $L$ and $\tilde{L}$. Namely, one can see easily that

$$
\mathbb{E}\left(z_{T}^{*}(t)^{\gamma+1}\right)=\mathbb{E}\left(\zeta_{t}^{* \gamma+1}\right) \mathbb{E}\left(\tilde{\zeta}^{*}{ }_{T-t}^{\gamma+1}\right)
$$

and that

$$
\mathbb{E}\left(z_{T}^{*}(t) \ln z_{T}^{*}(t)\right)=\mathbb{E}\left(\zeta_{t}^{*} \ln \zeta_{t}^{*}\right)+\mathbb{E}\left(\tilde{\zeta}_{T-t}^{*} \ln \tilde{\zeta}_{T-t}^{*}\right)
$$

In turn, the last quantities can be easily expressed via the corresponding Girsanov parameters using Ito formula.

Proof of Theorem 1 Since $\mathbb{Q}^{*}$ is an equivalent martingale measure we have from (14) that

$$
f\left(\mathbb{Q}_{T}^{*} \mid \mathbb{P}_{T}\right)=\mathbb{E}\left[f\left(c(\tau) \zeta_{\tau} \frac{\tilde{\zeta}_{T}}{\tilde{\zeta}_{\tau}}\right)\right]
$$

It follows from the independence of $L, \tilde{L}$ and $\tau$ that

$$
\mathbb{E}\left[f\left(c(\tau) \zeta_{\tau} \frac{\tilde{\zeta}_{T}}{\tilde{\zeta}_{\tau}}\right) \mid \tau=t\right]=\mathbb{E}\left[f\left(c(t) \zeta_{t} \frac{\tilde{\zeta}_{T}}{\tilde{\zeta}_{t}}\right)\right]
$$

Now, the independence of $L$ and $\tilde{L}$ implies the conditional independence of $\zeta$ and $\tilde{\zeta}$ given $\sigma\left(L_{s}, s \leq T\right)$. Using the convexity of $f$ and invariance of $f$ under scaling, we see that in order to minimize $f$-divergence, the measure $\mathbb{Q}$ should be such that $\zeta$ is 
the density of an $f$-minimal martingale measure for $\left(e^{L_{t}}\right)_{t \geq 0}$ and $\tilde{\zeta}$ the density of an $f$-minimal martingale measure for $\left(e^{\tilde{L}_{t}}\right)_{t \geq 0}$. Hence (17) holds.

To find $f$-minimal equivalent martingale measure we have to minimize the function

$$
F(c)=\int_{0}^{T} \mathbb{E}\left[f\left(c(t) z_{T}^{*}(t)\right)\right] d \alpha(t)
$$

over all cadlag functions $c:[0, T] \rightarrow \mathbb{R}^{+, *}$ such that $\mathbb{E} c(\tau)=1$. For that we consider the linear space $\mathcal{L}$ of such cadlag functions $c:[0 ; T] \rightarrow \mathbb{R}$ with the norm $\|c\|=$ $\sup _{t \in[0, T]}|c(t)|$ and also the cone of such positive functions.

We apply Kuhn-Tucker theorem (see [38]) to the function

$$
F_{\lambda}(c)=F(c)-\lambda \int_{0}^{T}(c(t)-1) d \alpha(t)
$$

with Lagrangian factor $\lambda>0$. We show that the Frechet derivative $\frac{\partial F_{\lambda}}{\partial c}$ of $F_{\lambda}(c)$, defined by

$$
\lim _{\|\delta\| \rightarrow 0} \frac{\left|F_{\lambda}(c+\delta)-F_{\lambda}(c)-\frac{\partial F_{\lambda}}{\partial c} \delta\right|}{\|\delta\|}=0
$$

is equal to:

$$
\frac{\partial F_{\lambda}}{\partial c}(\delta)=\int_{0}^{T}\left(\mathbb{E}\left[f^{\prime}\left(c(t) z_{T}^{*}(t)\right) z_{T}^{*}(t)\right]-\lambda\right) \delta(t) d \alpha(t)
$$

In fact, by the Taylor formula, we have for $\delta \in \mathcal{L}$ :

$$
\begin{gathered}
F_{\lambda}(c+\delta)-F_{\lambda}(c)-\frac{\partial F_{\lambda}}{\partial c} \delta= \\
\int_{0}^{T} \mathbb{E}\left[\left(f^{\prime}\left((c(t)+\theta(t)) z_{T}^{*}(t)\right)-f^{\prime}\left(c(t) z_{T}^{*}(t)\right)\right) z_{T}^{*}(t)\right] \delta(t) d \alpha(t)
\end{gathered}
$$

where $\theta(t)$ is a function which takes values in the interval $[0, \delta(t)]$. We remark that the modulus of the right-hand side in the previous equality is bounded from above by:

$$
\sup _{t \in[0, T]} \mathbb{E}\left[\left|f^{\prime}\left((c(t)+\theta(t)) z_{T}^{*}(t)\right)-f^{\prime}\left(c(t) z_{T}^{*}(t)\right)\right| z_{T}^{*}(t)\right]\|\delta\|
$$

and that hypothesis $\left(\mathcal{H}_{3}\right)$ implies that for all $c>0$

$$
\mathbb{E}\left[f^{\prime}\left(c z_{T}^{*}(t)\right) z_{T}^{*}(t)\right]<\infty
$$

Since $f^{\prime}$ is continuous and increasing and the functions $c$ and $\delta$ are bounded, we conclude by Lebesgue's dominated convergence theorem that (19) holds and then (20).

Then, in order to $\frac{\partial F_{\lambda}}{\partial c} \delta=0$ for all $\delta \in \mathcal{L}$, it is necessary and sufficient to take $c$ such that

$$
\mathbb{E}\left[f^{\prime}\left(c(t) z_{T}^{*}(t)\right) z_{T}^{*}(t)\right]-\lambda=0 \quad \alpha \text {-a.s. }
$$

Finally, for each $c>0$ and $t \in[0, T]$ we consider the function

$$
\lambda_{t}(c)=\mathbb{E}\left[f^{\prime}\left(c z_{T}^{*}(t)\right) z_{T}^{*}(t)\right] .
$$


We see easily that it is continuous and increasing in $c$ and that its right-continuous inverse $c_{t}(\lambda)$ satisfies:

$$
\lambda=\mathbb{E}\left[f^{\prime}\left(c_{t}(\lambda) z_{T}^{*}(t)\right) z_{T}^{*}(t)\right]
$$

Now, to obtain a minimizer $c^{*}$, it remains to find, if it exists, $\lambda^{*}$ which satisfies (18).

Let us consider now the special case $f^{\prime}(x)=a x^{\gamma}$. Then we obtain up to a constant, that $\lambda_{t}(c)=a c^{\gamma} \mathbb{E}\left[z_{T}^{*}(t)^{\gamma+1}\right]$ and for $f^{\prime}(x)=\ln (x)+1$ we get $\lambda_{t}(c)=\mathbb{E}\left[z_{T}^{*}(t) \ln z_{T}^{*}(t)\right]+1$. Finally, we write down $c_{t}(\lambda)$ and we integrate with respect to $\alpha$, and we find $\lambda^{*}$ and the expression of $c^{*}(t)$.

Example: A change-point Black-Scholes model. We apply the previous results when $L$ and $\tilde{L}$ define Black-Scholes type models. Therefore, we assume that $L$ and $\tilde{L}$ are continuous Levy processes with characteristics $(b, c, 0)$ and $(\tilde{b}, c, 0)$ respectively, $c>$ 0 . As is well known, the initial models will be complete, with a unique equivalent martingale measure which defines a unique price for options. However, in our changepoint model the martingale measure is not unique, and we have an infinite set of martingale measures of the form

$$
\frac{d \mathbb{Q}_{t}}{d \mathbb{P}_{t}}(X)=c(\tau) \exp \left(\int_{0}^{t} \beta_{s} d X_{s}^{c}-\frac{1}{2} \int_{0}^{t} \beta_{s}^{2} c d s\right)
$$

where $c(\cdot)$ is a measurable function $[0, T] \rightarrow \mathbb{R}^{+, *}$ such that $\mathbb{E}[c(\tau)]=1$ and

$$
\beta_{s}=-\frac{1}{c}\left[\left(b+\frac{c}{2}\right) \mathbf{1}_{\llbracket 0, \tau \rrbracket}(s)+\left(\tilde{b}+\frac{c}{2}\right) \mathbf{1}_{\rrbracket \tau,+\infty \llbracket}(s)\right]
$$

If for example $f^{\prime}(x)=a x^{\gamma}$, applying Theorem 1, we get

$$
c^{*}(t)=\frac{e^{-\frac{\gamma+1}{2 c}\left[\left(b+\frac{c}{2}\right)^{2} t+\left(\tilde{b}+\frac{c}{2}\right)^{2}(T-t)\right]}}{\int_{0}^{T} e^{-\frac{\gamma+1}{2 c}\left[\left(b+\frac{c}{2}\right)^{2} t+\left(\tilde{b}+\frac{c}{2}\right)^{2}(T-t)\right]} d \alpha(t)}
$$

and if $f^{\prime}(x)=\ln (x)+1$, then

$$
c^{*}(t)=\frac{e^{-\frac{1}{2 c}\left[\left(b+\frac{c}{2}\right)^{2} t+\left(\tilde{b}+\frac{c}{2}\right)^{2}(T-t)\right]}}{\int_{0}^{T} e^{-\frac{1}{2 c}\left[\left(b+\frac{c}{2}\right)^{2} t+\left(\tilde{b}+\frac{c}{2}\right)^{2}(T-t)\right]} d \alpha(t)}
$$

\section{Optimal strategies for utility maximization}

We start by recalling some useful basic facts about optimal strategies for utility maximization. Then some decomposition formulas will be given which permit us to find optimal strategies. We end up by giving the formulas for optimal strategies for utility maximization in change-point setting. 


\subsection{Some known facts}

In this subsection, we are interested in finding optimal strategies for terminal wealth with respect to some utility functions. More precisely, we assume that our financial market consists of two assets : a non-risky asset $B$, with interest rate $r$, and a risky asset $S$, modelled using the change-point Levy model defined in (4). We denote by $\vec{S}=(B, S)$ the price process and by $\vec{\Phi}=\left(\phi^{0}, \phi\right)$ the amount of money invested in each asset. According to usual terminology, a predictable $\vec{S}$-integrable process $\vec{\Phi}$ is said to be a self-financing admissible strategy if for every $t \in[0, T]$ and $x$ initial capital

$$
\vec{\Phi}_{t} \cdot \vec{S}_{t}=x+\int_{0}^{t} \vec{\Phi}_{u} \cdot d \vec{S}_{u}
$$

where the stochastic integral in the right-hand side is bounded from below. Here • denotes the scalar product. We will denote by $\mathcal{A}$ the set of all self-financing admissible strategies. In order to avoid unnecessary complications, we will assume that the interest rate $r$ is 0 , so that starting with an initial capital $x$, terminal wealth at time $T$ is

$$
V_{T}(\phi)=x+\int_{0}^{T} \phi_{s} d S_{s}
$$

Let $u$ denote a strictly increasing, strictly concave, continuously differentiable function on $\operatorname{dom}(u)=\{x \in \mathbb{R} \mid u(x)>-\infty\}$ which satisfies

$$
\begin{gathered}
u^{\prime}(+\infty)=\lim _{x \rightarrow+\infty} u^{\prime}(x)=0, \\
u^{\prime}(\underline{x})=\lim _{x \rightarrow \underline{x}} u^{\prime}(x)=+\infty
\end{gathered}
$$

where $\underline{x}=\inf \{u \in \operatorname{dom}(u)\}$.

We will say that $\phi^{*}$ defines an optimal strategy with respect to $u$ if

$$
\mathbb{E}_{P}\left[u\left(x+\int_{0}^{T} \phi_{s}^{*} d S_{s}\right)\right]=\sup _{\phi \in \mathcal{A}} \mathbb{E}_{P}\left[u\left(x+\int_{0}^{T} \phi_{s} d S_{s}\right)\right]
$$

As in [34], we will say that $\hat{\phi}$ is an asymptotically optimal strategy if there exists a sequence of stopping times $\left(\tau_{n}\right)_{n \in \mathbb{N}}$ which goes to infinity such that for each $n, \phi_{s}^{*} \mathbf{1}_{\llbracket 0, T \wedge \tau_{n} \rrbracket}$ is an admissible strategy for the restriction of $u$ on the interval $[-n,+\infty[$ and

$$
\lim _{n \rightarrow+\infty} E\left[u\left(x+\int_{0}^{T \wedge \tau_{n}} \hat{\phi}_{s} d S_{s}\right)\right]=\sup _{\phi \in \mathcal{A}} E\left[u\left(x+\int_{0}^{T} \phi_{s} d S_{s}\right)\right]
$$

As known, there is a strong link between this optimization problem and the previous problem of finding $f$-minimal martingale measures. Let $f$ be the convex conjugate function of $u$ :

$$
f(y)=\sup _{x \in \mathbb{R}}\{u(x)-x y\}=u(I(y))-y I(y)
$$


where $I=\left(u^{\prime}\right)^{-1}$. We recall that in particular

$$
\begin{aligned}
& \text { if } u(x)=\ln (x) \text { then } f(x)=-\ln (x)-1, \\
& \text { if } u(x)=\frac{x^{p}}{p}, p<1 \text { then } f(x)=-\frac{p-1}{p} x^{\frac{p}{p-1}}, \\
& \text { if } u(x)=1-e^{-x} \text { then } f(x)=1-x+x \ln (x) .
\end{aligned}
$$

The following result gives us the relation between portfolio optimization and f-minimal martingale measures.

Theorem 2. (cf. 27]) Let $x \in \mathbb{R}^{+}$be fixed. Let $Q^{*}$ be an equivalent martingale measure which satisfies

$$
\mathbb{E}_{P}\left|f\left(\lambda \frac{d Q_{T}^{*}}{d P_{T}}\right)\right|<\infty, \quad \mathbb{E}_{Q^{*}}\left|f^{\prime}\left(\lambda \frac{d Q_{T}^{*}}{d P_{T}}\right)\right|<\infty
$$

for $\lambda$ such that

$$
-\mathbb{E}_{Q^{*}} f^{\prime}\left(\lambda \frac{d Q_{T}^{*}}{d P_{T}}\right)=x
$$

Then, if $Q^{*}$ is an f-minimal martingale measure, there exists a predictable function $\phi^{*}$ such that $\left(\int_{0}^{*} \phi_{u}^{*} d S_{u}\right)$ is a $Q^{*}$-martingale and

$$
-f^{\prime}\left(\lambda \frac{d Q_{T}^{*}}{d P_{T}}\right)=x+\int_{0}^{T} \phi_{u}^{*} d S_{u}
$$

If the last relation holds, then $\vec{\Phi}=\left(\phi^{0}, \phi\right)$ with $\phi_{t}^{0}=x+\int_{0}^{t} \phi_{u} d S_{u}-\phi_{t} S_{t}$ is an asymptotically optimal portfolio strategy. Moreover, if $\underline{x}>-\infty$, this strategy is optimal.

Proof If $\underline{x}>-\infty$ the the result follows from [27], Theorem 3.1. If $\underline{x}=-\infty$ and (23) holds, then we show that there exists a sequence of stopping times $\left(\tau_{n}\right)_{n \in \mathbb{N}}$ which goes to infinity such that $\left(\phi_{s}^{*} \mathbf{1}_{\llbracket 0, T \wedge \tau_{n} \rrbracket}\right)_{0 \leq s \leq T}$ is a sequence of admissible strategies, and that $\phi^{*}$ is asymptotically optimal.

We put then

$$
\tau_{n}=\inf \left\{t \geq 0: \int_{0}^{t} \phi_{u}^{*} d S_{u} \leq-n\right\}
$$

It is obvious that the sequence of stopping times $\left(\tau_{n}\right)_{n \in \mathbb{N}^{*}}$ is going to infinity as $n \rightarrow \infty$ and

$$
\lim _{n \rightarrow+\infty} \mathbb{E}_{P}\left[u\left(x+\int_{0}^{T \wedge \tau_{n}} \phi_{s}^{*} d S_{s}\right)\right] \leq \sup _{\phi \in \mathcal{A}} \mathbb{E}_{P}\left[u\left(x+\int_{0}^{T} \phi_{s} d S_{s}\right)\right]
$$

Now, if $\phi$ is any admissible strategy, it follows from the concavity of $u$ that

$$
u\left(x+\int_{0}^{T} \phi_{s} d S_{s}\right) \leq u\left(x+\int_{0}^{T} \phi_{s}^{*} d S_{s}\right)+u^{\prime}\left(x+\int_{0}^{T} \phi_{s}^{*} d S_{s}\right)\left[\int_{0}^{T}\left(\phi_{s}-\phi_{s}^{*}\right) d S_{s}\right]
$$

Note that $u^{\prime}\left(x+\int_{0}^{T} \phi_{s}^{*} d S_{s}\right)=\zeta_{T}^{*}$ where $\zeta_{T}^{*}$ is Radon-Nikodym density of the measure $Q_{T}^{*}$ with respect to $P_{T}$. Note also that $\left(\int_{0}^{t}\left(\phi_{s}-\phi_{s}^{*}\right) d S_{s}\right)_{0 \leq t \leq T}$ is the difference of a local 
martingale which is bounded from below and of a martingale with respect to $Q^{*}$, so that

$$
\mathbb{E}_{P}\left[u\left(x+\int_{0}^{T} \phi_{s} d S_{s}\right)\right] \leq \mathbb{E}_{P}\left[u\left(x+\int_{0}^{T} \phi_{s}^{*} d S_{s}\right)\right]
$$

and hence

$$
\sup _{\phi \in \mathcal{A}} \mathbb{E}_{P}\left[u\left(x+\int_{0}^{T} \phi_{s} d S_{s}\right)\right] \leq \mathbb{E}_{P}\left[u\left(x+\int_{0}^{T} \phi_{s}^{*} d S_{s}\right)\right]
$$

Next, we have in the same way from the concavity of $u$ that

$$
u\left(x+\int_{0}^{T \wedge \tau_{n}} \phi_{s}^{*} d S_{s}\right) \leq u\left(x+\int_{0}^{T} \phi_{s}^{*} d S_{s}\right)+u^{\prime}\left(x+\int_{0}^{T} \phi_{s}^{*} d S_{s}\right) . \int_{T \wedge \tau_{n}}^{T} \phi_{s}^{*} d S_{s}
$$

Since $\left(\int_{0}^{T \wedge \tau_{n}} \phi_{s}^{*} d S_{s}\right)_{n \geq 1}$ is a uniformly integrable $Q^{*}$ martingale, the family $\left(\int_{T \wedge \tau_{n}}^{T} \phi_{s}^{*} d S_{s}\right)_{n \in \mathbb{N}}$ is uniformly integrable. Hence $\left(u\left(x+\int_{0}^{T \wedge \tau_{n}} \phi_{s}^{*} d S_{s}\right)\right)_{n \in \mathbb{N}}$ is a uniformly integrable family and

$$
\lim _{n \rightarrow+\infty} \mathbb{E}_{P}\left[u\left(x+\int_{0}^{T \wedge \tau_{n}} \phi_{s}^{*} d S_{s}\right)=\mathbb{E}_{P}\left[u\left(x+\int_{0}^{T} \phi_{s}^{*} d S_{s}\right)\right]\right.
$$

Finally, it follows from (24), (25) and (26) that $\phi^{*}$ defines an asymptotically optimal strategy.

\subsection{A decomposition formula}

We use the structure of $\mathbb{Q}^{*}$ presented in Theorem 1 to write down a decomposition formula mentioned in Theorem 2 for $f^{\prime}\left(\lambda Z_{T}^{*}(\tau)\right)$. First of all we give the expressions for Girsanov parameters when changing the measure $\mathbb{P}$ into $\mathbb{Q}^{*}$.

Lemma 1. Let Girsanov parameters of the $f$-divergence minimal equivalent martingale measures $Q^{*}$ and $\tilde{Q}^{*}$ are $\left(\beta^{*}, Y^{*}\right)$ and $\left.\tilde{\beta}^{*}, \tilde{Y}^{*}\right)$ respectively. Then the Girsanov parameters when changing from $\mathbb{P}$ to $\mathbb{Q}^{*}$ are:

$$
\begin{gathered}
\beta_{t}^{*}=\beta^{*} \mathbf{1}_{\llbracket 0, \tau \rrbracket}(t)+\tilde{\beta}^{*} \mathbf{1}_{\rrbracket \tau,+\infty \llbracket}(t) \\
Y_{t}^{*}=Y^{*} \mathbf{1}_{\llbracket 0, \tau \rrbracket}(t)+\tilde{Y}^{*} \mathbf{1}_{\rrbracket \tau,+\infty \llbracket}(t) .
\end{gathered}
$$

Next, we introduce for fixed $u \in[0, T], x \geq 0$ and $t \in[0, T]$ the quantities

$$
\rho^{(u)}(t, x)=\mathbb{E}\left(Z_{T}^{*}(\tau) f^{\prime}\left(Z_{T}^{*}(\tau)\right) \mid \tau=u, Z_{t}^{*}(u)=x\right)=\mathbb{E}\left(Z_{T}^{*}(u) f^{\prime}\left(Z_{T}^{*}(u)\right) \mid Z_{t}^{*}(u)=x\right)
$$

and we remark that the regular in $u$ and right-continuous versions of processes verify: $\mathbb{P}$-a.s. for all $t \in[0, T]$

$$
\mathbb{E}\left(Z_{T}^{*}(\tau) f^{\prime}\left(Z_{T}^{*}(\tau)\right) \mid \mathcal{F}_{t}\right)=\rho^{(\tau)}\left(t, Z_{t}^{*}(\tau)\right)
$$

To simplify the notation we introduce $\eta_{T-t}(u)$ such that

$$
\eta_{T-t}(u)=\frac{z_{T}^{*}(u)}{z_{t}^{*}(u)}
$$


and we remark that

$$
\eta_{T-t}(u) \stackrel{\mathcal{L}}{=} z_{T-t}^{*}\left((u-t)^{+}\right) .
$$

As a consequence of previous formulas, we have

$$
\rho^{(u)}(t, x)=\mathbb{E}\left[\eta_{T-t}(u) f^{\prime}\left(x \eta_{T-t}(u)\right)\right]
$$

Now, we would like to use Ito formula for $\rho^{(u)}\left(t, Z_{t}^{*}(u)\right)$. But the mentioned function is not sufficiently smooth and we will proceed by approximations. For that we construct a sequence of functions $\left(\phi_{n}\right)_{n \geq 1}$.

Lemma 2. Let $f$ be convex function belonging to $C^{3}\left(\mathbb{R}^{+, *}\right)$. There exists a sequence of bounded functions $\left(\phi_{n}\right)_{n \geq 1}$, which are of class $\mathcal{C}^{2}$ on $\mathbb{R}^{+, *}$, increasing, such that for all $n \geq 1, \phi_{n}$ coincides with $f^{\prime}$ on the compact set $\left[\frac{1}{n}, n\right]$ and such that for sufficiently big $n$ the following inequalities hold for all $x, y>0$ :

$$
\left|\phi_{n}(x)\right| \leq 4\left|f^{\prime}(x)\right|+\alpha,\left|\phi_{n}^{\prime}(x)\right| \leq 3 f^{\prime \prime}(x),\left|\phi_{n}(x)-\phi_{n}(y)\right| \leq 5\left|f^{\prime}(x)-f^{\prime}(y)\right|
$$

where $\alpha$ is a real positive constant.

Proof We set, for $n \geq 1$,

$$
\begin{gathered}
A_{n}(x)=f^{\prime}\left(\frac{1}{n}\right)-\int_{x \vee \frac{1}{2 n}}^{\frac{1}{n}} f^{\prime \prime}(y)(2 n y-1)^{2}(5-4 n y) d y \\
B_{n}(x)=f^{\prime}(n)+\int_{n}^{x \wedge(n+1)} f^{\prime \prime}(y)(n+1-y)^{2}(1+2 y-2 n) d y
\end{gathered}
$$

and finally

$$
\phi_{n}(x)=\left\{\begin{array}{l}
A_{n}(x) \text { if } 0 \leq x<\frac{1}{n} \\
f^{\prime}(x) \text { if } \frac{1}{n} \leq x \leq n \\
B_{n}(x) \text { if } x>n
\end{array}\right.
$$

Proof. We can verify easily that $\phi_{n}$ coincide with $f^{\prime}$ on $\left[\frac{1}{n}, n\right]$ and that the properties (28) hold.

Now we introduce

$$
\rho_{n}^{(u)}(t, x)=\mathbb{E}\left(Z_{T}^{*}(\tau) \phi_{n}\left(Z_{T}^{*}(\tau)\right) \mid \tau=u, Z_{t}^{*}(u)=x\right)
$$

and we see that

$$
\rho_{n}^{(u)}\left(t, Z_{T}^{*}(u)\right)=\mathbb{E}\left[\eta_{T-t}(u) \phi_{n}\left(x \lambda \eta_{T-t}(u)\right)\right]
$$

In the next lemma we give a decomposition formula for $\rho_{n}^{(u)}$. For that we put

$$
\xi_{t}^{(n, u)}(x)=\mathbb{E}\left[\eta_{T-t}(u) \phi_{n}^{\prime}\left(x \eta_{T-t}(u)\right)\right]
$$

and

$$
H_{t}^{(n, u)}(x, y)=\mathbb{E}\left(\eta_{T-t}(u)\left[\phi_{n}\left(x \eta_{T-t}(u) Y_{t}^{*}(y)\right)-\phi_{n}\left(x \eta_{T-t}(u)\right)\right]\right)
$$

and we denote $Q_{u}^{*}$ the conditional law $Q^{*}$ given $\tau=u$. 
Lemma 3. We have $\mathbb{Q}_{u}^{*}$-a.s., for all $t \leq T$,

$$
\begin{gathered}
E_{\mathbb{Q}_{u}^{*}}\left[\phi_{n}\left(Z_{T}^{*}(u)\right) \mid \mathcal{G}_{t}\right]=E_{\mathbb{Q}_{u}^{*}}\left[\phi_{n}\left(Z_{T}^{*}(u)\right)\right]+ \\
\int_{0}^{t} \beta_{s}^{*} Z_{s-}^{*}(u) \xi_{s}^{(n, u)}\left(Z_{s-}^{*}(u)\right) d X_{s}^{(c), \mathbb{Q}_{u}^{*}}+\int_{0}^{t} \int_{\mathbb{R}} H_{s}^{(n, u)}\left(Z_{s-}^{*}(u), y\right)\left(\mu^{X}-\nu^{X, \mathbb{Q}_{u}^{*}}\right)(d s, d y)
\end{gathered}
$$

where $\nu^{X, \mathbb{Q}_{u}^{*}}$ is a compensator of the jump measure $\mu^{X}$ with respect to $\left(\mathbb{F}, \mathbb{Q}_{u}^{*}\right)$.

Proof In order to apply the Ito formula to $\rho_{n}^{(u)}$, we show that $\rho_{n}$ is twice continuously differentiable with respect to $x$ and once with respect to $t$ on the set $x \geq \epsilon, \epsilon>0$ and $t \in[0, T]$ and that the corresponding derivatives are bounded. Then we apply the Ito formula to $\rho_{n}^{(u)}$ but stopped at stopping times

$$
s_{m}=\inf \left\{t \geq 0 \mid Z_{t}^{*}(u) \leq \frac{1}{m}\right\}
$$

with $m \geq 1$ and $\inf \{\emptyset\}=\infty$.

From strong Markov property of Levy processes we have:

$$
\rho_{n}^{(u)}\left(t \wedge s_{m}, Z_{t \wedge s_{m}}^{*}(u)\right)=E_{\mathbb{Q}_{u}^{*}}\left(\phi_{n}\left(Z_{T}^{*}(u)\right) \mid \mathcal{G}_{t \wedge s_{m}}\right)
$$

and we remark that $\left(E_{\mathbb{Q}_{u}^{*}}\left(\phi_{n}\left(Z_{T}^{*}(u)\right) \mid \mathcal{G}_{t \wedge s_{m}}\right)\right)_{t \geq 0}$ is a $\mathbb{Q}_{u}^{*}$ - martingale. By Ito formula we obtain that:

$$
\begin{aligned}
& \rho_{n}^{(u)}(t \wedge\left.s_{m}, Z_{t \wedge s_{m}}^{*}(u)\right)=\rho_{n}^{(u)}\left(0, Z_{0}^{*}(u)\right)+\int_{0}^{t \wedge s_{m}} \frac{\partial \rho_{n}^{(u)}}{\partial s}\left(s, Z_{s-}^{*}(u)\right) d s+ \\
& \int_{0}^{t \wedge s_{m}} \frac{\partial \rho_{n}^{(u)}}{\partial x}\left(s, Z_{s-}^{*}(u)\right) d Z_{s-}^{*}(u)+\frac{1}{2} \int_{0}^{t \wedge s_{m}} \frac{\partial^{2} \rho_{n}^{(u)}}{\partial x^{2}}\left(s, Z_{s-}^{*}(u)\right) d\left\langle Z^{*, c}(u)\right\rangle_{s}+ \\
& \int_{0}^{t \wedge s_{m}} \int_{\mathbb{R}}\left(\rho_{n}\left(s, Z_{s-}^{*}(u)+x\right)-\rho_{n}\left(s, Z_{s-}^{*}(u)\right)-\frac{\partial \rho_{n}}{\partial x}\left(s, Z_{s-}^{*}(u)\right) x\right) \mu^{Z^{*}}(d s, d x)
\end{aligned}
$$

Then we can write that

$$
\rho_{n}^{(u)}\left(t \wedge s_{m}, Z_{t \wedge s_{m}}^{*}(u)\right)=A_{t \wedge s_{m}}+M_{t \wedge s_{m}}
$$

with

$$
\begin{gathered}
A .=\int_{0}^{\cdot} \frac{\partial \rho_{n}^{(u)}}{\partial s}\left(s, Z_{s-}^{*}(u)\right) d s+\frac{1}{2} \int_{0}^{\cdot} \frac{\partial^{2} \rho_{n}^{(u)}}{\partial x^{2}}\left(s, Z_{s-}^{*}(u)\right) d\left\langle Z^{*, c}(u)\right\rangle_{s}+ \\
\int_{0} \int_{\mathbb{R}}\left[\rho_{n}^{(u)}\left(s, Z_{s-}^{*}(u)+x\right)-\rho_{n}^{(u)}\left(s, Z_{s-}^{*}(u)\right)-\frac{\partial \rho_{n}^{(u)}}{\partial x}\left(s, Z_{s-}^{*}(u)\right) x\right] \nu^{Z^{*}, \mathbb{Q}_{u}^{*}}(d s, d x) \text { and } \\
M=\int_{0}^{\cdot} \frac{\partial \rho_{n}^{(u)}}{\partial x}\left(s, Z_{s-}^{*}(u)\right) d Z_{s-}^{*}(u)+ \\
\int_{0} \int_{\mathbb{R}}\left[\rho_{n}^{(u)}\left(s, Z_{s-}^{*}(u)+x\right)-\rho_{n}^{(u)}\left(s, Z_{s-}^{*}(u)\right)\left(\mu^{Z^{*}}(d s, d x)-\nu^{Z^{*}, \mathbb{Q}_{u}^{*}}(d s, d x)\right)\right.
\end{gathered}
$$


But since $A$ is predictable process and $\left(E_{\mathbb{Q}_{u}^{*}}\left(\phi_{n}\left(Z_{T}^{*}(u)\right) \mid \mathcal{G}_{t \wedge s_{m}}\right)\right)_{t \geq 0}$ is a $\mathbb{Q}_{u^{-}}^{*}$ martingale, we obtain that $\mathbb{Q}_{u}^{*}$-a.s., $A_{t}=0$ for all $0 \leq t \leq T$.

From [49], corollary 2.4, p. 59, we get since $\sigma\left(\cup_{m=1}^{\infty} \mathcal{G}_{t \wedge s_{m}}\right)=\mathcal{G}_{t}$ that

$$
\lim _{m \rightarrow \infty} \rho_{n}^{(u)}\left(t \wedge s_{m}, Z_{t \wedge s_{m}}^{*}(u)\right)=E_{\mathbb{Q}^{*}}\left(\phi_{n}\left(Z_{T}^{*}(u)\right) \mid \mathcal{G}_{t}\right)
$$

Moreover, we remark that for all $x \in \mathbb{R}$ and $s \in[0, T]$

$$
\frac{\partial \rho_{n}^{(u)}}{\partial x}(s, x)=\xi_{s}^{(n, u)}(x)
$$

and all $x, y \in \mathbb{R}$ and $s \in[0, T]$

$$
H_{s}^{(n, u)}(x, y)=\rho_{n}^{(u)}\left(s, x Y^{*}(y)\right)-\rho_{n}^{(u)}(s, x)
$$

We conclude using the definition of local martingales that the decomposition of Lemma holds.

The next step consists to pass to the limit in previous decomposition. For that let us denote for $0 \leq t \leq T$

$$
\xi_{t}^{(u)}(x)=\mathbb{E}\left[\eta_{T-t}(u) f^{\prime \prime}\left(x \eta_{T-t}(u)\right)\right]
$$

and

$$
H_{t}^{(u)}(x, y)=\mathbb{E}\left(\eta_{T-t}(u)\left[f^{\prime}\left(x \eta_{T-t}(u) Y_{t}^{*}(y)\right)-f^{\prime}\left(x \eta_{T-t}(u)\right)\right]\right)
$$

Lemma 4. We have $\mathbb{Q}_{u}^{*}$-a.s., for all $t \leq T$,

$$
\begin{gathered}
E_{\mathbb{Q}_{u}^{*}}\left(f^{\prime}\left(Z_{T}^{*}(u)\right) \mid \mathcal{G}_{t}\right)=E_{\mathbb{Q}_{u}^{*}}\left[f^{\prime}\left(Z_{T}^{*}(u)\right)\right]+ \\
\int_{0}^{t} \beta_{s}^{*} Z_{s-}^{*}(u) \xi_{s}^{(u)}\left(Z_{s-}(u)\right) d X_{s}^{(c), \mathbb{Q}_{u}^{*}}+\int_{0}^{t} \int_{\mathbb{R}} H_{s}^{(u)}\left(Z_{s-}(u), y\right)\left(\mu^{X}-\nu^{X, \mathbb{Q}_{u}^{*}}\right)(d s, d y)
\end{gathered}
$$

where $\nu^{X, \mathbb{Q}_{u}^{*}}$ is a compensator of the jump measure $\mu^{X}$ with respect to $\left(\mathbb{F}, \mathbb{Q}_{u}^{*}\right)$.

Proof. The proof consists to show the convergence in probability of stochastic integrals and conditional expectations using the properties of $\phi_{n}$ cited in Lemma 2 and can be performed in the same way as in [6].

\subsection{Optimal strategies in a change-point situation}

Let $u$ be a utility function belonging to $C^{3}(] \underline{x},+\infty[)$ and $f$ its convex conjugate, $f \in$ $C^{3}\left(\mathbb{R}^{+, *}\right)$. We suppose that $\mathcal{M}(P) \neq \emptyset$ and $\mathcal{M}(\tilde{P}) \neq \emptyset$ and we introduce the following hypotheses

$\left(\mathcal{H}_{4}\right)$ : For each compact set $K$ of $\mathbb{R}^{+, *}$ and $t \in[0, T]$, we have:

$$
\sup _{\lambda \in K} \sup _{t \in[0, T]} E_{Q^{*}}\left[\zeta_{t}^{*} f^{\prime \prime}\left(\lambda \zeta_{t}^{*}\right)\right]<\infty, \sup _{\lambda \in K} \sup _{t \in[0, T]} E_{\tilde{Q}^{*}}\left[\tilde{\zeta}_{t}^{*} f^{\prime \prime}\left(\lambda \tilde{\zeta}_{t}^{*}\right)\right]<\infty
$$

where $\zeta^{*}$ and $\tilde{\zeta}^{*}$ are the densities of the $f$-minimal equivalent martingale measures $Q^{*}$ and $\tilde{Q}^{*}$ with respect to $P$ and $\tilde{P}$ respectively. 
Theorem 3. Let $u$ be a strictly concave function belonging to $C^{3}(] \underline{x},+\infty[)$. Suppose that a convex conjugate $f$ of $u$ satisfy $\left(\mathcal{H}_{1}\right),\left(\mathcal{H}_{2}\right),\left(\mathcal{H}_{3}\right),\left(\mathcal{H}_{4}\right)$ and $(18)$. Then there exists an $\mathbb{F}$-optimal strategy $\phi^{*}$ for change-point model (4). In addition, it is $\hat{\mathbb{F}}$-adapted for $f(x)=x \ln (x)$. If $c \neq 0$, then

$$
\phi_{t}^{*}=-\frac{\beta_{t}^{*} Z_{t-}^{*}(\tau)}{S_{t-}} \xi\left(t, Z_{t-}^{*}(\tau)\right)
$$

with $\beta^{*}$ defined in Lemma 1 .

If $c=0$ and $\operatorname{supp}(\nu) \neq \emptyset$, supp $(\tilde{\nu}) \neq \emptyset$ both containing 0 , then $f^{\prime \prime}(x)=$ ax $x^{\gamma}$ with $a>0$ and $\gamma \in \mathbb{R}$, and the optimal strategies are defined by the same formula but with the replacement of $\beta_{t}^{*}$ by $\alpha_{t}^{*}$ such that

$$
\alpha_{t}^{*}=Y^{*}\left(y_{0}\right)^{\gamma} \frac{\partial Y^{*}}{\partial y}\left(y_{0}\right) \mathbf{1}_{\{\tau>t\}}+\tilde{Y}^{*}\left(y_{1}\right)^{\gamma} \frac{\partial \tilde{Y}^{*}}{\partial y}\left(y_{1}\right) \mathbf{1}_{\{\tau \leq t\}}
$$

where $y_{0} \in \operatorname{sipp}(\nu)$ and $y_{1} \in \operatorname{suipp}(\tilde{\nu})$.

Proof From Theorem 1 and the hypotheses $\left(\mathcal{H}_{1}\right),\left(\mathcal{H}_{2}\right),\left(\mathcal{H}_{3}\right)$ and $(18)$ it follows that there exists an $f$-minimal martingale measure $\mathbb{Q}^{*}$. Since the processes $X$ and $S$ are $\hat{\mathbb{F}}$-adapted, applying for example Theorem 3.1 in [27], we have the existence of an $\hat{\mathbb{F}}$-adapted optimal strategy $\phi^{*}$ such that

$$
-f^{\prime}\left(\lambda Z_{T}^{*}(\tau)\right)=x+\int_{0}^{T} \phi_{u}^{*} d S_{u}
$$

and such that $\int_{0} \phi_{u}^{*} d S_{u}$ defines a local martingale with respect to $(\mathbb{Q}, \hat{\mathbb{F}})$. The same is true for initially enlarged filtration $\mathbb{F}$. Then, for $c \neq 0$, we compare the decomposition of Lemma 4 and the decomposition of Theorem 2 to get our formulas. For $c=0$ we use first the Theorem 3 of [5] to prove that $f^{\prime \prime}(x)=a x^{\gamma}$. Then, again we compare the decomposition of Lemma 4 and the decomposition of Theorem 2 to get our formulas.

Proposition 2. Let $u$ be common utility function and let $f$ be its convex conjugate, $f^{\prime \prime}(x)=a x^{\gamma}$, where $a>0$ and $\gamma \in \mathbb{R}$. Suppose that $c \neq 0$ or $\operatorname{supp}(\nu) \neq \emptyset$, supp $(\tilde{\nu}) \neq \emptyset$ and both containing 0 . Then there exists an u-asymptotically optimal strategy if and only if there exist $\alpha, \beta \in \mathbb{R}^{d}$ and measurable function $Y: \mathbb{R} \backslash\{0\} \rightarrow \mathbb{R}^{+, *}$ such that

$$
Y(y)=\left(f^{\prime}\right)^{-1}\left(f^{\prime}(1)+\alpha\left(e^{y}-1\right)\right)
$$

and such that the following properties hold:

$$
\begin{gathered}
Y(y)>0 \nu-\text { a.e. } \\
\int_{|y| \geq 1}\left(e^{y}-1\right) Y(y) \nu(d y)<+\infty . \\
b+\frac{1}{2} c+c \beta+\int_{\mathbb{R}^{d}}\left(\left(e^{y}-1\right) Y(y)-l(y)\right) \nu(d y)=0 .
\end{gathered}
$$


and the same conditions are verified with the replacement of $\alpha, \beta$ by $\tilde{\alpha}, \tilde{\beta}, \nu$ by $\tilde{\nu}$ and $Y$ by $\tilde{Y}$. Furthermore, if $c \neq 0$ then

$$
\phi_{t}^{*}=-\frac{\beta_{t}^{*}\left(Z_{t-}^{*}(\tau)\right)^{\gamma+1}}{S_{t-}} \mathbb{E}\left(\left[z_{T-t}^{*}\left((\tau-t)^{+}\right)\right]^{\gamma+1} \mid \tau\right)
$$

with $\beta_{t}^{*}=\beta I_{\llbracket 0, \tau \rrbracket}(t)+\tilde{\beta} I_{\rrbracket \tau,+\infty \llbracket}(t)$.

If $c=0$ and $\operatorname{supp}(\nu) \neq \emptyset$, supp $(\tilde{\nu}) \neq \emptyset$ both containing 0 , then

$$
\phi_{t}^{*}=-\frac{\alpha_{t}\left(Z_{t-}^{*}(\tau)\right)^{\gamma+1}}{S_{t-}} \mathbb{E}\left(\left[z_{T-t}^{*}\left((\tau-t)^{+}\right)\right]^{\gamma+1} \mid \tau\right)
$$

with $\alpha_{t}^{*}$ given in Theorem 3. In addition, $\phi^{*}$ is optimal as soon as $\gamma \neq-1$. Moreover, $\phi^{*}$ is $\hat{\mathbb{F}}$-adapted as soon as $\gamma=-1$.

Proof We recall from [5] that under the assumptions (37), (38) and (39), the Levy model associated with $L$ has an $f$-minimal equivalent martingale measure which preserves the Levy property and whose Girsanov parameters are $\beta$ and $Y$. The same is true for the Levy model associated with $\tilde{L}$. Then, the formulas for strategies follow directly from Theorem 3, $\square$

Example: Optimal strategy for Black-Scholes model with change point and exponential utility. As before, we now want to apply the results when $L$ and $\tilde{L}$ define Black-Scholes type models. Therefore, we assume that $L$ and $\tilde{L}$ are continuous Levy processes with characteristics $(b, c, 0)$ and $(\tilde{b}, c, 0)$ respectively. Let $\tau$ be a random variable bounded by $T$ which is independent from $L$ and $\tilde{L}$. Then the asymptotically optimal strategy from the point of view of maximization of exponential utility $u(x)=1-\exp (-x)$ will be :

$$
\phi_{t}^{*}=-\frac{\beta_{t}}{S_{t-}}=\frac{(b+c / 2) \mathbf{1}_{\llbracket 0, \tau \rrbracket}(t)+(\tilde{b}+c / 2) \mathbf{1}_{\rrbracket \tau,+\infty \llbracket}(t)}{c S_{t-}}
$$

\section{Acknowledgements}

This work is supported in part by ECOS project M07M01 and by ANR-09-BLAN0084-01 of the Department of Mathematics of Angers's University.

\section{References}

[1] S. Ankirchner, C. Blanchet-Scalliet, A. Eyraud-Losel Credit risk premium and quadratic BSDE's With a single jump. Working paper.

[2] M. Basseville, I. V. Nikiforov. Detection of Abrupt changes-Theory and Applications. Prentice Hall, Englewood Cliffs, NJ, 1993.

[3] E. Bayraktar, S. Dayanik, I. Karatzas. The standard Poisson disorder problem revisited. Stoch. proc. Appl. 115, 9 (2005) 1437-1450. 
[4] P. Carr, H. Geman, D. Madan, M. Yor. Stochastic volatility for Levy processes. Mathematical Finance, 13, 2 ( 2003) 345-382.

[5] S. Cawston, L. Vostrikova. Levy preservation and associated properties for $f$-divergence minimal equivalent martingale measures. Working paper.

[6] S. Cawston, L. Vostrikova. f-divergence approach and optimal portfolios in exponential Levy models. Working paper.

[7] A. Cerny, J. Kallsen. On the structure of general mean-variance hedging strategies. Cass Business School Research Paper. Available at SSRN: http://ssrn.com/abstract=882762.

[8] J. Chen, A. Gupta. Statistical inference of covariance change points in Gaussian model. Statistics 38,1 (2004) 17-28.

[9] J. Chen, A. Gupta. Testing and Locating Variance changepoint with Application to Stock prices. Journal of the American Statistical Association 92 (1997)

[10] X. Chen, J. Wan. Option pricing for Time-change Exponential levy Model under MEMM. Acta Mathematicae Applicatae Sinica, English Series, 23, 4 (2007) 651-664

[11] T. Choulli, C. Stricker. Minimal entropy-Hellinger martingale measure in incomplete markets. Mathematical Finance, 15 ( 2005) 465-490.

[12] T. Choulli, C. Stricker, J. Li. Minimal Hellinger martingale measures of order q. Finance Stoch. 11,3 (2007) 399-427.

[13] CzIsar I. Information-type measure of divergence of probability distributions. MTA Oztaly Kezlemenyei 17 (1987) 123-149, 267-299.

[14] M. H. A. Davis. A note on the Poisson disorder problem. Banach Center Publ. 1 (1976) 65-72.

[15] A. Dias, P. Embrechts. Change-point analysis for dependence structures in finance and insurance. In: Risk Measures for the 21st Century, ed. by Giorgio Szegoe, Wiley Finance Series, 321-335, 2004.

[16] E. Eberlein. Jump-type Levy processes. In Handbook of Financial Series. Springer-Verlag, 2007.

[17] N. El Karoui, M. Jeanblanc, Ying Jiao What happens after a default: The conditional density approach. Stochastic processes and their applications. 120 (2010) 1011-1032.

[18] F. Esche, M. Schweizer. Minimal entropy preserves the Lévy property : how and why. Stochastic processes and their applications, 115, 2 (2005) 299-327. 
[19] H. Follmer, M. Schweizer. Hedging of Contingent Claims under Incomplete information. in: M.H. Davis and R.J. Elliott(eds), "Applied Stochastic Analysis", Stochastics Monographs, vol.5, Gordon and Breach, London/New York (1991) 389414 .

[20] T. Fujinara, Y. Miyahara. The Minimal Entropy Martingale Measures for Geometric Levy Processes. Finance and Stochastics. 7 (2003) 509-531.

[21] L. J. Galchuk, B. V. Rozovsky. The disorder problem for a Poisson process. Theory probab. Appl. 16 (1971) 729-734.

[22] A. Gandy, U. Jensen, C. Lutkebohmert. A Cox model with a change-point Applied to an Actuarial Problem. Braz. J. Probab. Stat. 19 (2005) 93 -109.

[23] P. V. Gapeev, G. Pechkir The Wiener disorder problem with finite horizon. Stoch. stoch. Rep.76 , 1 (2004) 59-75.

[24] Gasbarra D., Valkeila E., Vostrikova L. Enlargement of filtration and additional information in pricing models: Bayesian approach. In Kabanov Yu., Liptser R., Stoyanov D. From Stochastic Calculus to Mathematical Finance, 257-285, Springer-Verlag, 2006.

[25] H. Geman, D. Madan, M. Yor Time changes for Levy processes. Mathematical Finance, 11, 1 (2001) 79-96.

[26] H. Geman, D. Madan, M. Yor Stochastic volatility, jumps and hidden time changes. Finance Sthoch. 6 (2002) 63-90.

[27] T. Goll, L. Ruschendorf. Minimax and minimal distance martingale measures and their relationship to portfolio optimization. Finance and Stochastics, Vol. V.4 (2001) 557-581.

[28] P. Grandits. On martingale measures for stochastic processes with independent increments. Theory Probab. Appl. vol.44, 1 (1999) 39-50.

[29] X. Guo. A regime switching model: Statistical estimation, empirical evidence, and change point detection, Proc. SIAM-AMS-IMA Research Conference in Mathematical Finance, 139-155, 2004.

[30] D.M. Hawkins, K. D. Zamba A change-Point Model for a Shift in Variance. Journal of Quality Technology, 37, 1 (2005) 21-31.

[31] F. Hubalek, C. Sgarra. Esscher transforms and the minimal entropy martingale measure for exponential Levy models Quantitative finance, 6, 2 (2006) 125-145.

[32] J. Jacod, A. Shiryaev. Limit Theorems for Stochastic Processes. SpringerVerlag, 1987. 
[33] M. Jeanblanc, S. Kloppel., Y. Miyahara. Minimal $F^{Q}$-martingale measures for exponential Levy processes. Ann. Appl. Probab. 17, 5/6 (2007), 1615-1638.

[34] J. Kallsen. Optimal portfolios for exponential Lévy processes. Mathematical Methods of Operations Research, 51 (2000) 357-374.

[35] J. Kallsen. Utility-based derivative pricing in incomplete markets. In H. Geman, D. Madan, S. Pliska, and T. Vorst, editors, Mathematical Finance - Bachelier Congress 2000, 313-338, Berlin, Springer 2002.

[36] J. Kallsen, A. Shiryaev. The cumulant process and Esscher's change of measure. Finance and Stochastics, 6 (2002) 397-428.

[37] T. Kavtaradze , N. Lazrieva, M. Mania, P. Muliere. A Bayesianmartingale approach to the general disorder problem. Stochastic processes Appl. 117 (2007 )8, 1093-1120.

[38] R. KitTer Duality for Nonlinear Programming in a Banach Space. SIAM Journal on Applied Mathematics, 15, 2 ( 1967) 294-302.

[39] N. Lazrieva, Thoronjadze Optimal robust mean-variance hedging in incomplete financial markets. Journal of Mathematical Sciences 153, 3(2008) 262-290

[40] F. Liese, I. Vajda. Convex statistical distances, Teubner Texte zur Mathematik 95. Teubner Verl. Leipzig ,1986.

[41] A. Lokka Detection of disorder before an observable event. Preprint, 24/10/2006.

[42] Y. Mryahara. Minimal Entropy Martingale Measures of Jump Type Price Process in Incomplete Assets Markets. Asian-Pacific Financial Markets. 6, 2 (1999) 97-113.

[43] Y. Miyahara, A. Novikov. Geometric Levy Process Pricing Model. Proceedings of Steklov Mathematical Institute, 237 (2002) 176-191.

[44] E. S. PAGE. A test for a change in parameter occuring at an unknown point. biometrika, 42 (1955) 523-527.

[45] E. S. PAGE. On problems in which a change in parameter occurs at an unknown point Biometrika, 44 (1957) 248-252.

[46] G. Peskir, A. N. Shiryaev. Solving the Poisson disorder problem. Advance in finance and stochastics, Springer, Berlin, (2002) 295-312.

[47] M. Pollak, D. Siegmund. A diffusion process and its applications to detecting a change in the drift of Brownian motion. Bometrika, 72, 2 (1985) 267-280.

[48] S. W. RoberTs. A comparison of some control chart procedures. Technometrics, 8 (1966) 411-430. 
[49] D.Revuz, M.Yor Continuous Martingales and Brownian Motion, SpringerVerlag, Berlin, 1999.

[50] K. Sato. Levy processes and infinitely divisible distributions CUP (1999).

[51] M. Schweizer. On the minimal martingale measure and the Follmer-Schweizer decomposition. Stochastic Analysis and Applications, 13 (1995), 573-599.

[52] M. Schweizer. A Guided Tour through Quadratic Hedging approaches, In: E. Jouini, J. Cvitanic, M. Musiela (eds.) :"Option Pricing, Interest Rates and Risk Management", Cambridge University Press, (1999) 538-574.

[53] A. N. Shiryaev. On optimum methods in quickest detection problems. Theory Probab. Appl., 8 (1963) 22-46.

[54] A. Shiryaev. Essentials of Stochastic Finance. World Scientific, Singapore, 1999.

[55] A. N. Shiryaev. From "Disorder" to Nonlinear Filtering and Martingale theory. Mathematical events in the twentieth century, Springer, Berlin, 2006.

[56] A. N. ShiRYAEv. Quickest detection problems in the technical analysis of financial data. Mathematical Finance-Bachelier Congress, 2000 (Paris), 487-521, Springer Finance, Springer, Berlin.

[57] A. N. Shiryaev. On Stochastic models and optimal methods in the problems of the quickest detection, Theory of probability and their applications,53, 3 (2009) 385-401.

[58] V. G. Spokoiny. On sequential detection of a small disorder. Frontiers in Pure and Applied Probab. 1, 238-255, H. Niemi et al. (Eds) 1993 TVP/VSP.

[59] S. Sukparungsee, A. Novikov. On EWMA procedure for detection of a change in observations via martingale approach. KMITL Sci. J. 6, 2 (2006) 373-380. 


\title{
$F$-divergence minimal equivalent martingale measures and optimal portfolios for exponential Levy models with a change-point
}

\author{
October 4, 2018
}

S. Cawston] and L. Vostrikova²

\begin{abstract}
We study exponential Levy models with change-point which is a random variable, independent from initial Levy processes. On canonical space with initially enlarged filtration we describe all equivalent martingale measures for changepoint model and we give the conditions for the existence of f-divergence minimal equivalent martingale measure. Using the connection between utility maximisation and $f$-divergence minimisation, we obtain a general formula for optimal strategy in change-point case for initially enlarged filtration and also for progressively enlarged filtration in the case of exponential utility. We illustrate our results considering the Black-Scholes model with change-point.

KEY WORDS AND PHRASES: f-divergence, exponential Levy models, changepoint, optimal portfolio

MSC 2010 subject classifications: 60G46, 60G48, 60G51, 91B70
\end{abstract}

\section{Introduction}

The parameters of financial models are generally highly dependent on time : a number of events (for example the release of information in the press, changes in the price of raw materials or the first time a stock price hits some psychological level) can trigger a change in the behaviour of stock prices. This time-dependency of the parameters can often be described using a piece-wise constant function : we will call this case a change-point model. In this context, an important problem in financial mathematics will be option pricing and hedging. Of course, the time of change (change-point) for

\footnotetext{
1,2 LAREMA, Département de Mathématiques, Université d'Angers, 2, Bd Lavoisier - 49045, Angers Cedex 01.

${ }^{1}$ E-mail: suzanne.cawston@univ-angers.fr ${ }^{2}$ E-mail: lioudmila.vostrikova@univ-angers.fr
} 
the parameters is not explicitly known, but it is often possible to make reasonable assumptions about its nature and use statistical tests for its detection.

Change-point problems have a long history, probably beginning with the papers of Page [45], 46] in an a-posteriori setting, and of Shiryaev [54] in a quickest detection setting. The problem was later considered in many papers, see for instance [14], [49], [21], 48], 33], 59], 42] and also the book [2] and references there. In the context of financial mathematics, the question was investigated in [31], [8], [30], [22], [15], [57], [58], 38], 60] and was often related to a quickest detection approach.

It should be noticed that not only quickest detection approach is interesting in financial mathematics, and this fact is related with pricing and hedging of so called default models (see [1], [17] and references there). In mentioned papers a number of very important results was obtained but for the processes without jump part or with only one jump.

The models with jumps, like exponential Levy models, in general, compromise the uniqueness of an equivalent martingale measure when such measure exists. So, one has to choose in some way an equivalent martingale measure to price. Many approaches have been developed and various criteria suggested for this choice of martingale measure, for example risk-minimization in an $L^{2}$-sense [40] [19], [52], [53], Hellinger integrals minimization [11], [12], 28], entropy minimization [43], 20], [18], $f^{q}$-martingale measures [34] or Esscher measures 32].

All these approaches can be considered in unified way using so called $f$-divergences, introduced by Ciszar [13] and investigated in a number of papers and books (see for instance [41] and references there). It should also be noticed that a general characterisation of $f$-divergence minimal martingale measures with applications to exponential Levy models was given first in [27].

We recall that for $f$ a convex function on $\mathbb{R}^{+, *}$ and two measures $Q$ and $P$ such that $Q<<P$, the $f$-divergence of $Q$ with respect to $P$ is defined as

$$
f(Q \mid P)=\mathbb{E}_{P}\left[f\left(\frac{d Q}{d P}\right)\right]
$$

where $\frac{d Q}{d P}$ is Radon-Nikodym density of $Q$ with respect to $P$, and $E_{P}$ is the expectation with respect to $P$. We recall that the utility maximisation is closely related to $f$-divergence minimisation via Fenchel-Legendre transform and this will be one of essential points to obtain an optimal strategy.

The aim of this paper is to study $f$-divergence minimal martingale measures and optimal portfolios from the point of view of utility maximization, for exponential Levy model with change-point where the parameters of the model before and after the change are known and a change-point itself is a random variable, independent from initial Levy processes. We remark that even complete models like Black-Scholes model, become to be incomplete in change-point setting. This is why simple conditioning with respect to $\tau$ and the use of the results on the processes with independent increments can not give us a right answer immediately.

We start by describing our model in more details. We assume the financial market 
consists of a non-risky asset $B$ with interest rate $\left(r_{t}\right)_{t \geq 0}$, namely

$$
B_{t}=B_{0} \exp \left(\int_{0}^{t} r_{s} d s\right)
$$

where

$$
r_{t}=r \mathbf{1}_{\{\tau>t\}}+\tilde{r} \mathbf{1}_{\{\tau \leq t\}},
$$

with $r, \tilde{r}$ interest rates before and after change-point $\tau$, and a one-dimensional risky asset $S=\left(S_{t}\right)_{t \geq 0}$,

$$
S_{t}=S_{0} \exp \left(X_{t}\right)
$$

where $X$ is a stochastic process obtained by pasting in $\tau$ of two Levy processes $L$ and $\tilde{L}$ together:

$$
X_{t}=L_{t} \mathbf{1}_{\{\tau>t\}}+\left(L_{\tau}+\tilde{L}_{t}-\tilde{L}_{\tau}\right) \mathbf{1}_{\{\tau \leq t\}}
$$

Here and further $L$ and $\tilde{L}$ supposed to be independent Levy processes with characteristics $(b, c, \nu)$ and $(\tilde{b}, \tilde{c}, \tilde{\nu})$ respectively which are independent from $\tau$ ( for more details see [51]). To avoid unnecessary complications we assume up to now that for change-point model $r$ and $\tilde{r}$ in (2) are equal to zero, and that $S_{0}=1$.

To describe a probability space on which the process $X$ is well-defined, we consider $(D, \mathcal{G}, \mathbb{G})$ the canonical space of right-continuous functions with left-hand limits equipped with its natural filtration $\mathbb{G}=\left(\mathcal{G}_{t}\right)_{t \geq 0}$ which satisfies standard conditions: it is right-continuous, $\mathcal{G}_{0}=\{\emptyset, D\}, \bigvee_{t \geq 0} \mathcal{G}_{t}=\mathcal{G}$. On the product of such canonical spaces we define two independent Levy processes $L=\left(L_{t}\right)_{t \geq 0}$ and $\tilde{L}=\left(\tilde{L}_{t}\right)_{t \geq 0}$ with characteristics $(b, c, \nu)$ and $(\tilde{b}, \tilde{c}, \tilde{\nu})$ respectively and denote by $P$ and $\tilde{P}$ their respective laws which are assumed to be locally equivalent: $P \stackrel{\text { loc }}{\sim} \tilde{P}$. As we will consider the market on a fixed finite time interval, we are really only interested in $\left.P\right|_{\mathcal{G}_{T}}$ and $\left.\tilde{P}\right|_{\mathcal{G}_{T}}$ for a fixed $T \geq 0$ and the distinction between equivalence and local equivalence does not need to be made.

Our change-point will be represented by an independent random variable $\tau$ of law $\alpha$ taking values in $([0, T], \mathcal{B}([0, T])$. The set $\{\tau=T\}$ corresponds to the situation when the change-point does not take place, or at least not on the interval we are studying.

On the probability space $(D \times D \times[0, T], \mathcal{G} \times \mathcal{G} \times \mathcal{B}([0, T], P \times \tilde{P} \times \alpha)$ we define a measurable map $X$ by (4) and we denote by $\mathbb{P}$ its law. In what follows we use $\mathbb{E}$ mainly for the expectation with respect to $\mathbb{P}$ but this notation will be also used for the expectation with respect to $P \times \tilde{P} \times \alpha$.

From point of view of observable processes we can have the following situations. If we observe only the process $X$ then the natural probability space to work is $(D, \mathcal{G}, \mathbb{P})$ equipped with the right-continuous version of the natural filtration $\mathbb{G}=\left(\mathcal{G}_{t}\right)_{t \geq 0}$ where $\mathcal{G}_{t}=\sigma\left\{X_{s}, s \leq t\right\}$ for $t \geq 0$. Now, if we observe not only the process $X$ but also some complementary variables related with $\tau$ then we can take it in account by the enlargement of the filtration. First we consider the filtration $\mathbb{H}$ given by $\mathcal{H}_{t}=\sigma\left(\mathbf{1}_{\{\tau \leq s\}}, s \leq t\right)$ and note that $\mathcal{H}_{T}=\sigma(\tau)$. Then we introduce two filtrations: the initially enlarged filtration $\mathbb{F}=\left(\mathcal{F}_{t}\right)_{t \geq 0}$

$$
\mathcal{F}_{0}=\mathcal{G}_{0} \vee \mathcal{H}_{T}, \quad \mathcal{F}_{t}=\bigcap_{s>t}\left(\mathcal{G}_{s} \vee \mathcal{H}_{T}\right)
$$


and the progressively enlarged filtration $\hat{\mathbb{F}}=\left(\hat{\mathcal{F}}_{t}\right)_{t \geq 0}$ which satisfies :

$$
\hat{\mathcal{F}}_{0}=\mathcal{G}_{0} \vee \mathcal{H}_{0}, \quad \hat{\mathcal{F}}_{t}=\bigcap_{s>t}\left(\mathcal{G}_{s} \vee \mathcal{H}_{s}\right)
$$

In the case of additional information the most natural filtration from the point of view of observable events would be $\hat{\mathbb{F}}$. However, it is not so easy to obtain the explicit formulas of optimal strategies for progressively enlarged filtration. So, we start by investigation of optimal strategies for initially enlarged filtration. In a special case of exponential utility it gives us an optimal strategy for progressively enlarged filtration. Our approach applied for density process with respect to progressively enlarged filtration gives also answer on optimal strategy for logarithmic utility, but a general case is still form an open question.

The paper is organized in the following way. In 2 . we start by recalling in unified way the facts about f-divergence minimal equivalent martingale measures for exponential Levy models. This information will be used for investigation of change-point case.

In 3 we investigate the change-point case. On mentioned probability space and for initially enlarged filtration we describe first all equivalent martingale measures. Then, we introduce as hypotheses, such properties of $f$-divergence minimal equivalent martingale measures as a preservation of Levy property and a scaling property. The question of preservation of Levy property was considered in details in [5] and it was shown that the class of $f$-divergences preserving Levy property is larger then common $f$-divergences, i.e. the functions such that $f^{\prime \prime}(x)=a x^{\gamma}, a>0, \gamma \in \mathbb{R}$. We recall that these functions are those for which there exists $A>0$ and real $B, C$ such that $f(x)=A f_{\gamma}(x)+B x+C$ where

$$
f_{\gamma}(x)=\left\{\begin{array}{l}
c_{\gamma} x^{\gamma+2} \text { if } \gamma \neq-1,-2 \\
x \ln (x) \text { if } \gamma=-1 \\
-\ln (x) \text { if } \gamma=-2
\end{array}\right.
$$

and $c_{\gamma}=\operatorname{sign}[(\gamma+1)(\gamma+2)]$. The conditions for existence and the expression of RadonNikodym density $Z_{T}^{*}(\tau)$ of $f$-divergence minimal martingale measure for change-point model is given in Theorem 2. Then, in Corollaries 4 and 5 we give the corresponding results for common f-divergences and, finally, we apply the results to Black-Scholes change-point model.

In 4. we present first some facts about utility maximisation and the formulas for optimal strategies of single exponential Levy model. Then we give a decomposition formula for $f^{\prime}\left(Z_{T}^{*}(\tau)\right)$ for initially enlarged filtration. These decompositions allow us via the result of [27] to identify optimal strategy (see Theorem 5). We illustrate these results by considering again the Black-Scholes model with a change-point. 


\section{$2 \quad f$-divergence minimal EMM's for exponential Levy model}

We start by recalling in unified way the facts about $f$-divergence minimal martingale measures for exponential Levy models. Namely, we will consider common $f$-divergences and we will discuss the preservation of Levy property by $f$-divergence minimal locally equivalent martingale measures (EMM's), we will mention the expressions for so called Girsanov parameters when we change the initial measure $P$ into $f$-divergence minimal EMM's and also the expression of Radon-Nikodym density of these measures via Girsanov parameters.

Let now $L=\left(L_{t}\right)_{t \geq 0}$ be Levy process with parameters $(b, c, \nu)$ where $b$ is the drift parameter, $c$ is the diffusion parameter and $\nu$ is the Levy measure, i.e. the measure on $\mathbb{R} \backslash\{0\}$ which satisfies

$$
\int_{\mathbb{R}}\left(x^{2} \wedge 1\right) \nu(d x)<+\infty .
$$

We recall that the characteristic function of $L_{t}$ for $t \in \mathbb{R}^{+}$and $u \in \mathbb{R}$ is given then by:

$$
\phi_{t}(u)=\mathbb{E} e^{i u L_{t}}=e^{\psi(u) t}
$$

and in turn, the characteristic exponent

$$
\psi(u)=i u b-\frac{1}{2} c u^{2}+\int_{\mathbb{R}}(\exp (i u x)-1-i u h(x)) \nu(d x),
$$

where from now on, $h$ is the truncation function. We set $S=\left(S_{t}\right)_{t \geq 0}$ with

$$
S_{t}=S_{0} \exp \left(L_{t}\right)
$$

for our risky asset and $B=\left(B_{t}\right)_{t \geq 0}$ for non-risky asset with constant interest rate $r$. We will suppose without loss of generality up to now that $S_{0}=1$ and $r=0$.

Let $T$ be a fixed horizon and $\mathbb{G}=\left(\mathcal{G}_{t}\right)_{t \geq 0}$ be natural filtration. We recall that for a convex function $f$ on $\mathbb{R}^{+, *}$, the $f$-divergence of the restriction $Q_{T}$ of the measure $Q$ with respect to the restriction $P_{T}$ of the measure $P$ to $\mathcal{G}_{T}$ is:

$$
f\left(Q_{T} \mid P_{T}\right)=E_{P}\left[f\left(\frac{d Q_{T}}{d P_{T}}\right)\right]
$$

Here by convention we set this integral equal to $+\infty$ if the corresponding function is not integrable. We recall that $Q_{T}^{*}$ is an $f$-divergence minimal equivalent martingale measure if $f\left(Q_{T}^{*} \mid P_{T}\right)<+\infty$ and

$$
f\left(Q_{T}^{*} \mid P_{T}\right)=\inf _{Q \in \mathcal{M}(P)} f\left(Q_{T} \mid P_{T}\right)
$$

where $\mathcal{M}(P)$ is the set of locally equivalent martingale measures supposed to be nonempty. We also recall that an $f$-divergence minimal equivalent martingale measure $Q^{*}$ is invariant under scaling if for all $x \in \mathbb{R}^{+, *}$

$$
f\left(x Q_{T}^{*} \mid P_{T}\right)=\inf _{Q \in \mathcal{M}(P)} f\left(x Q_{T} \mid P_{T}\right)
$$


It is called time-invariant if $Q^{*}$ is the same for all $T>0$. For a given exponential Levy model $S=S_{0} e^{L}$, we say that an $f$-divergence minimal martingale measure $Q^{*}$ preserves the Levy property if $L$ remains a Levy process under $Q^{*}$.

We recall that the density $Z$ of any equivalent to $P$ measure $Q$ can be written in the form $Z=\mathcal{E}(M)$ where $\mathcal{E}$ denotes the Doleans-Dade exponential and $M=\left(M_{t}\right)_{t \geq 0}$ is a local martingale. It follows from Girsanov theorem that there exist predictable functions $\beta$ and $Y$ verifying the following integrability conditions : for $t \geq 0$ ( $P$-a.s.)

$$
\begin{gathered}
\int_{0}^{t} \beta_{s}^{2} d s<\infty \\
\int_{0}^{t} \int_{\mathbb{R}}\left|h(y)\left(Y_{s}(y)-1\right)\right| \nu^{X, P}(d s, d y)<\infty,
\end{gathered}
$$

and such that

$$
M_{t}=\int_{0}^{t} \beta_{s} d X_{s}^{c}+\int_{0}^{t} \int_{\mathbb{R}}\left(Y_{s}(y)-1\right)\left(\mu^{X}-\nu^{X, P}\right)(d s, d y)
$$

where $\mu^{X}$ is a jump measure of the process $X$ and $\nu^{X, P}$ is its compensator with respect to $(P, \mathbb{G}), \nu^{X, P}(d s, d y)=d s \nu(d y)$ ( for more details see [33]). We will refer to $(\beta, Y)$ as the Girsanov parameters of the change of measure from $P$ into $Q$. It is known from Grigelionis result [29] that a semi-martingale is a process with independent increments under $Q$ if and only if their semi-martingale characteristics are deterministic, i.e. the Girsanov parameters do not depend on $\omega$, i.e. $\beta$ depends only on time $t$ and $Y$ depends on $(t, x)$ time and jump size. Since Levy process is homogeneous process, it implies that $X$ will remain a Levy process under $Q$ if and only if there exists $\beta \in \mathbb{R}$ and a positive measurable function $Y$ such that for all $t \leq T$ and all $\omega, \beta_{t}(\omega)=\beta$ and $Y_{t}(\omega, y)=Y(y)$.

We recall that if Levy property is preserved, $S$ will be a martingale under $Q$ if and only if

$$
b+\frac{1}{2} c+c \beta+\int_{\mathbb{R}}\left[\left(e^{y}-1\right) Y(y)-h(y)\right] \nu(d y)=0
$$

This follows again from Girsanov theorem and reflects the fact that under $Q$ the drift of $S$ is equal to zero.

As it was mentioned, our aim in this section is to consider in more detail the class of minimal martingale measures for the functions which satisfy (77). In particular, the minimal measure for $f$ will be the same as that for $f_{\gamma}$. Minimal measures for the different functions $f_{\gamma}$ have been well studied (see [27] and further references). It has been shown in [35], [18], 34] that in all these cases, the minimal measure, when it exists, preserves the Levy property.

Sufficient conditions for the existence of a minimal measure and an explicit expression of the associated Girsanov parameters have been given in the case of relative entropy in [20], 32] and for power functions in [34. It was also shown in [32] that these conditions are in fact necessary in the case of relative entropy or for power functions. Our aim in this section is to give a unified expression of such conditions for all functions 
which satisfy $f^{\prime \prime}(x)=a x^{\gamma}$ and to show that, under some conditions, they are necessary and sufficient. We have already mentioned that $f$-divergence minimal martingale measures play an important role in the determination of utility maximising strategies. In this context, it is useful to have further invariance properties for the minimal measures such as scaling and time invariance properties. This is the case when $f^{\prime \prime}(x)=a x^{\gamma}$.

Theorem 1. Consider a Levy process $X$ with characteristics $(b, c, \nu)$ and let $f$ be $a$ function such that $f^{\prime \prime}(x)=a x^{\gamma}$, where $a>0$ and $\gamma \in \mathbb{R}$. Suppose that $c \neq 0$ or supp $(\nu) \neq \emptyset$. Then there exists an $f$-divergence minimal equivalent to $P$ martingale measure $Q$ preserving Levy properties if and only if there exist constants $\alpha, \beta \in \mathbb{R}$ and measurable function $Y: \mathbb{R} \backslash\{0\} \rightarrow \mathbb{R}^{+}$such that

$$
Y(y)=\left(f^{\prime}\right)^{-1}\left(f^{\prime}(1)+\alpha\left(e^{y}-1\right)\right)
$$

and such that the following properties hold:

$$
\begin{gathered}
Y(y)>0 \nu-\text { a.e. } \\
\int_{|y| \geq 1}\left(e^{y}-1\right) Y(y) \nu(d y)<+\infty . \\
b+\frac{1}{2} c+c \beta+\int_{\mathbb{R}}\left(\left(e^{y}-1\right) Y(y)-h(y)\right) \nu(d y)=0 .
\end{gathered}
$$

If such a measure exists the Girsanov parameters associated with $Q$ are: $(\beta, Y)$ if $c \neq 0$, and $(0, Y)$ if $c=0$. In addition, this measure is scale and time invariant.

We begin with some technical lemmas. For $Q \stackrel{\text { loc }}{\sim} P$ we denote by $\left(Z_{t}\right)_{t \geq 0}$ RadonNikodym density process of $Q$ with respect to $P$.

Lemma 1. Let $Q$ be the measure preserving Levy property. Then, $Q_{T} \sim P_{T}$ for all $T>0$ iff

$$
\begin{gathered}
Y(y)>0 \nu-\text { a.e. } \\
\int_{\mathbb{R}}(\sqrt{Y(y)}-1)^{2} \nu(d y)<+\infty .
\end{gathered}
$$

Proof See Theorem 2.1, p. 209 of [33].

Lemma 2. Under $Q_{T} \sim P_{T}$, the condition $E_{P}\left|f\left(Z_{T}\right)\right|<\infty$ is equivalent to

$$
\int_{\mathbb{R}}\left[f(Y(y))-f(1)-f^{\prime}(1)(Y(y)-1)\right] \nu(d y)<+\infty
$$


Proof In our particular case, $E_{P}\left|f\left(Z_{T}\right)\right|<\infty$ is equivalent to the existence of $E_{P} f\left(Z_{T}\right)$. We use Ito formula to express this integrability condition in predictable terms. Taking for $n \geq 1$ stopping times

$$
s_{n}=\inf \left\{t \geq 0: Z_{t}>n \text { or } Z_{t}<1 / n\right\}
$$

where $\inf \{\emptyset\}=+\infty$, we get for $\gamma \neq-1,-2$ and $\alpha=\gamma+2$ that $P$-a.s.

$$
\begin{gathered}
Z_{T \wedge s_{n}}^{\alpha}=1+\int_{0}^{T \wedge s_{n}} \alpha \beta Z_{s-}^{\alpha} d X_{s}^{c}+\int_{0}^{T \wedge s_{n}} \int_{\mathbb{R}} Z_{s-}^{\alpha}\left(Y^{\alpha}(y)-1\right)\left(\mu^{X}-\nu^{X, P}\right)(d s, d y) \\
+\frac{1}{2} \alpha(\alpha-1) \beta^{2} c \int_{0}^{T \wedge s_{n}} Z_{s-}^{\alpha} d s+\int_{0}^{T \wedge s_{n}} \int_{\mathbb{R}} Z_{s-}^{\alpha}\left[Y^{\alpha}(y)-1-\alpha(Y(y)-1)\right] d s \nu(d y)
\end{gathered}
$$

Hence,

$$
Z_{T \wedge s_{n}}^{\alpha}=\mathcal{E}\left(N^{(\alpha)}+A^{(\alpha)}\right)_{T \wedge s_{n}}
$$

where

$$
N_{t}^{(\alpha)}=\int_{0}^{t} \alpha \beta d X_{s}^{c}+\int_{0}^{t}\left(Y^{\alpha}(y)-1\right)\left(\mu^{X}-\nu^{X, P}\right)(d s, d y)
$$

and

$$
A_{t}^{(\alpha)}=\frac{t}{2} \alpha(\alpha-1) \beta^{2} c+t \int_{\mathbb{R}}\left[Y^{\alpha}(y)-1-\alpha(Y(y)-1)\right] \nu(d y)
$$

Since $\left[N^{(\alpha)}, A^{(\alpha)}\right]_{t}=0$ for each $t \geq 0$ we have

$$
Z_{T \wedge s_{n}}^{\alpha}=\mathcal{E}\left(N^{(\alpha)}\right)_{T \wedge s_{n}} \mathcal{E}\left(A^{(\alpha)}\right)_{T \wedge s_{n}}
$$

In the case $\alpha>1$ and $\alpha<0$, and $E_{P} Z_{T}^{\alpha}<\infty$, we have by Jensen inequality

$$
0 \leq Z_{T \wedge s_{n}}^{\alpha} \leq E_{P}\left(Z_{T}^{\alpha} \mid \mathcal{F}_{T \wedge s_{n}}\right)
$$

and since the right-hand side of this inequality form uniformly integrable sequence, $\left(Z_{T \wedge s_{n}}^{\alpha}\right)_{n \geq 1}$ is also uniformly integrable. We remark that $A_{t}^{(\alpha)} \geq 0$ for all $t \geq 0$ and

$$
\mathcal{E}\left(A^{(\alpha)}\right)_{T \wedge s_{n}}=\exp \left(A_{T \wedge s_{n}}^{(\alpha)}\right) \geq 1 .
$$

It means that $\left(\mathcal{E}\left(N^{(\alpha)}\right)_{T \wedge s_{n}}\right)_{n \in \mathbb{N}^{*}}$ is uniformly integrable and

$$
E_{P}\left(Z_{T}^{\alpha}\right)=\exp \left(A_{T}^{(\alpha)}\right)
$$

If (17) holds, then by Fatou lemma and since $\mathcal{E}\left(N^{(\alpha)}\right)$ is a local martingale we get

$$
E_{P}\left(Z_{T}^{\alpha}\right) \leq \underline{\lim }_{n \rightarrow \infty} E_{P}\left(Z_{T \wedge s_{n}}\right) \leq \exp \left(A_{T}^{(\alpha)}\right)<\infty
$$

For $0<\alpha<1$, we have again

$$
Z_{T \wedge s_{n}}^{\alpha}=\mathcal{E}\left(N^{(\alpha)}\right)_{T \wedge s_{n}} \mathcal{E}\left(A^{(\alpha)}\right)_{T \wedge s_{n}}
$$


with uniformly integrable sequence $\left(Z_{T \wedge s_{n}}^{\alpha}\right)_{n \geq 1}$. Since

$$
\mathcal{E}\left(A^{(\alpha)}\right)_{T \wedge s_{n}}=\exp \left(A_{T \wedge s_{n}}^{(\alpha)}\right) \geq \exp \left(A_{T}^{(\alpha)}\right),
$$

the sequence $\left(\mathcal{E}\left(N^{(\alpha)}\right)_{T \wedge s_{n}}\right)_{n \in \mathbb{N}^{*}}$ is uniformly integrable and

$$
E_{P}\left(Z_{T}^{\alpha}\right)=\exp \left(A_{T}^{(\alpha)}\right)
$$

For $\gamma=-2$ we have that $f(x)=x \ln (x)$ up to linear term and

$$
\begin{gathered}
Z_{T \wedge s_{n}} \ln \left(Z_{T \wedge s_{n}}\right)= \\
\int_{0}^{T \wedge s_{n}}\left(\ln \left(Z_{s-}\right)+1\right) Z_{s-} \beta d X_{s}^{c}+\int_{0}^{T \wedge s_{n}} \int_{\mathbb{R}} Z_{s-}\left[\ln \left(Z_{s-}\right)(Y(y)-1)+Y(y) \ln (Y(y)]\left(\mu^{X-\nu^{X}, P}\right)(d s, d y)\right. \\
+\frac{1}{2} \beta^{2} c \int_{0}^{T \wedge s_{n}} Z_{s-} d s+\int_{0}^{T \wedge s_{n}} \int_{\mathbb{R}} Z_{s-}(Y(y) \ln (Y(y))-Y(y)+1) d s \nu(d y)
\end{gathered}
$$

Taking mathematical expectation we obtain:

$$
E_{P}\left[Z_{T \wedge s_{n}} \ln \left(Z_{T \wedge s_{n}}\right)\right]=E_{P} \int_{0}^{T \wedge s_{n}} Z_{s-}\left[\frac{1}{2} \beta^{2} c+\int_{\mathbb{R}}(Y(y) \ln (Y(y))-Y(y)+1) \nu(d y)\right] d s
$$

If $E_{P}\left[Z_{T} \ln \left(Z_{T}\right)\right]<\infty$, then the sequence $\left(Z_{T \wedge s_{n}} \ln \left(Z_{T \wedge s_{n}}\right)\right)_{n \in \mathbb{N}^{*}}$ is uniformly integrable. In addition, $E_{P}\left(Z_{s-}\right)=1$ and we obtain applying Lebesgue convergence theorem that

$$
E_{P}\left[Z_{T} \ln \left(Z_{T}\right)\right]=T\left[\frac{1}{2} \beta^{2} c+\int_{\mathbb{R}}(Y(y) \ln (Y(y))-Y(y)+1) \nu(d y)\right]
$$

and this implies (17). If (17), then by Fatou lemma from (21) we deduce that $E_{P}\left[Z_{T} \ln \left(Z_{T}\right)\right]<\infty$. For $\gamma=-1$, we have $f(x)=-\ln (x)$ and exchanging $P$ and $Q$ we get:

$$
E_{P}\left[-\ln \left(Z_{T}\right)\right]=E_{Q}\left[\tilde{Z}_{T} \ln \left(\tilde{Z}_{T}\right)\right]=T\left[\frac{1}{2} \beta^{2} c+\int_{\mathbb{R}}(\tilde{Y}(y) \ln (\tilde{Y}(y))-\tilde{Y}(y)+1) \nu^{Q}(d y)\right]
$$

where $\tilde{Z}_{T}=1 / Z_{T}$ and $\tilde{Y}(y)=1 / Y(y)$. But $\nu^{Q}(d y)=Y(y) \nu(d y)$ and, finally,

$$
E_{P}\left[-\ln \left(Z_{T}\right)\right]=\frac{T}{2} \beta^{2} c+T \int_{\mathbb{R}}(-\ln (Y(y))+Y(y)-1) \nu(d y)
$$

which implies (17). Again by Fatou lemma we get from (17) that $E_{P}\left[-\ln \left(Z_{T}\right)\right]<\infty$

Lemma 3. If the second Girsanov parameter $Y$ has a particular form (11) then the condition

$$
\int_{|y| \geq 1}\left(e^{y}-1\right) Y(y) \nu(d y)<+\infty
$$

implies the conditions (16) and (17). 
Proof We can cut each integral in (16) and (17) on two parts and integrate on the sets $\{|y| \leq 1\}$ and $\{|y|>1\}$. Then we can use a particular form of $Y$ and conclude easily writing Taylor expansion of order 2 .

Proof of Theorem 1 Necessity We suppose that there exist $f$-divergence minimal equivalent martingale measure $Q$ preserving Levy property of $X$. Then, since $Q_{T} \sim P_{T}$, the conditions (43), (16) follow from Theorem 2.1, p. 209 of [33]. From Theorem 3 of [5] we deduce that (11) holds. Then, the condition (44) follows from the fact that $S$ is a martingale under $Q$. Finally, the condition (45) follows from Girsanov theorem since $Q$ is a martingale measure and, hence, the drift of $S$ under $Q$ is zero.

Sufficiency We take $\beta$ and $Y$ verifying the conditions (43),(44),(45) and we construct

$$
M_{t}=\int_{0}^{t} \beta d X_{s}^{c}+\int_{0}^{t} \int_{\mathbb{R}}(Y(y)-1)\left(\mu^{X}-\nu^{X, P}\right)(d s, d y)
$$

As known from Theorem 1.33, p.72-73, of [33], the last stochastic integral is well defined if

$$
\begin{aligned}
& C(W)=T \int_{\mathbb{R}}(Y(y)-1)^{2} I_{\{|Y(y)-1| \leq 1\}} \nu(d y)<\infty, \\
& C\left(W^{\prime}\right)=T \int_{\mathbb{R}}|Y(y)-1| I_{\{|Y(y)-1|>1\}} \nu(d y)<\infty .
\end{aligned}
$$

But the condition (44), the relation (11) and Lemma 3 implies (16). So, $(Y-1) \in$ $G_{l o c}\left(\mu^{X}\right)$ and $M$ is local martingale. Then we take

$$
Z_{T}=\mathcal{E}(M)_{T}
$$

and this defines the measure $Q_{T}$ by its Radon-Nikodym density. Now, the conditions (43), (44) together with the relation (11) and Lemma 3 imply (16), and, hence, from Lemma 1 we deduce that $P_{T} \sim Q_{T}$.

Since $P_{T} \sim Q_{T}$, the Lemma2 2 gives us the needed integrability condition: $E_{P}\left|f\left(Z_{T}\right)\right|<\infty$. Now, since (45) holds, $Q$ is martingale measure, and it remains to show that $Q$ is indeed $f$-divergence minimal. For that we take any equivalent martingale measure $\bar{Q}$ and we show that

$$
E_{Q} f^{\prime}\left(Z_{T}\right) \leq E_{\bar{Q}} f^{\prime}\left(Z_{T}\right) .
$$

If the mentioned inequality holds, the Theorem 2.2 of [27] implies that $Q$ is an $f$ divergence minimal.

In the case $\gamma \neq-1,-2$ we obtain from (18) replacing $\alpha$ by $\gamma+1$ :

$$
Z_{T}^{\gamma+1}=\mathcal{E}\left(N^{(\gamma+1)}\right)_{T} \exp \left(A_{T}^{(\gamma+1)}\right)
$$

and using a particular form of $f^{\prime}$ and $Y$ we get that for $0 \leq t \leq T$

$$
N_{t}^{(\gamma+1)}=(\gamma+1) \beta \hat{X}_{t}
$$

where $\hat{X}$ is a stochastic logarithm of $S$. So, $\mathcal{E}\left(N^{(\gamma+1)}\right)$ is a local martingale and we get

$$
E_{\bar{Q}} Z_{T}^{\gamma+1} \leq \exp \left(A_{T}^{(\gamma+1)}\right)=E_{Q} Z_{T}^{\gamma+1}
$$


and, hence, (26).

In the case $\gamma=-1$ we prove using again a particular form of $f^{\prime}$ and $Y$ that

$$
f^{\prime}\left(Z_{T}\right)=E_{Q}\left(f^{\prime}\left(Z_{T}\right)\right)+\beta \hat{X}_{T}
$$

Since $E_{\bar{Q}} \hat{X}_{T} \leq 0$ we get that

$$
E_{\bar{Q}} f^{\prime}\left(Z_{T}\right) \leq E_{Q} f^{\prime}\left(Z_{T}\right)
$$

and it proves that $Q$ is $f$-divergence minimal.

The case $\gamma=-2$ can be considered in similar way.

Finally, note that the conditions which appear in Theorem 1 do not depend in any way on the time interval which is considered and, hence, the minimal measure always exists and its Girsanov parameters does not depend on $T$. So, the measure $Q^{*}$ is time invariant. Furthermore, if $Q^{*}$ is $f$-divergence minimal, the equality

$$
f(c x)=A f(x)+B x+C
$$

with $A, B, C$ constants, $A>0$, gives

$$
E_{P}\left[f\left(c \frac{d \bar{Q}}{d P}\right)\right]=A E_{P}\left[f\left(\frac{d \bar{Q}}{d P}\right)\right]+B+C \geq A E_{P}\left[f\left(\frac{d Q}{d P}\right)\right]+B+C=E_{P}\left[f\left(c \frac{d Q}{d P}\right)\right]
$$

and $Q$ is scale invariant.

Corollary 1. The existence of $f$-divergence EMM for power function $f(x)=c_{\gamma} x^{\gamma+2}$, $\gamma>-1$ or $\gamma<-2$ is equivalent to the existence of a strictly positive minimizer $Y$ for the integral

$$
I_{1}(\beta, Y)=\frac{1}{2}(\gamma+2)(\gamma+1) \beta^{2} c+\int_{\mathbb{R}}\left(Y^{\gamma+2}(y)-1-(\gamma+2)(Y(y)-1)\right) \nu(d y)
$$

under the constraint (45) over the set

$$
\mathcal{K}_{1}=\left\{(\beta, Y)\left|\beta \in \mathbb{R}, Y \geq 0, \int_{\mathbb{R}}\right|\left(e^{y}-1\right) Y(y)-h(y) \mid \nu(d y)<\infty, I_{1}(\beta, Y)<\infty\right\}
$$

For $-1<\gamma<-2$ it is equivalent to the existence of a strictly positive maximizer $Y$ for the same integral over the same $\mathcal{K}$. The solution to these optimisation problems can be only of the form:

$$
Y^{*}(y)= \begin{cases}\left(1+(\gamma+1) \beta^{*}\left(e^{y}-1\right)\right)^{\frac{1}{\gamma+1}} & \text { if } 1+(\gamma+1) \beta^{*}\left(e^{y}-1\right) \geq 0, \\ 0 & \text { in opposite case }\end{cases}
$$

with $\beta^{*}$ satisfying (45). 
Corollary 2. For the function $f(x)=x \ln (x)$, the existence of $f$-divergence EMM is equivalent to the existence of a strictly positive minimizer $Y$ for the integral

$$
I_{2}(\beta, Y)=\frac{1}{2} \beta^{2} c+\int_{\mathbb{R}}(Y(y) \ln (Y(y))-Y(y)+1) \nu(d y)
$$

under the constraint (45) over the set

$$
\mathcal{K}_{2}=\left\{(\beta, Y)\left|\beta \in \mathbb{R}, Y \geq 0, \int_{\mathbb{R}}\right|\left(e^{y}-1\right) Y(y)-h(y) \mid \nu(d y)<\infty, I_{2}(\beta, Y)<\infty\right\}
$$

The solution to this optimisation problems can be only of the form:

$$
Y(y)=\exp \left(\beta^{*}\left(e^{y}-1\right)\right)
$$

with $\beta^{*}$ satisfying 45 .

Corollary 3. For the function $f(x)=-\ln (x)$, the existence of $f$-divergence EMM is equivalent to the existence of a strictly positive minimizer $Y$ for the integral

$$
I_{3}(\beta, Y)=\frac{1}{2} \beta^{2} c+\int_{\mathbb{R}}(-\ln (Y(y))+Y(y)-1) \nu(d y)
$$

under the constraint (45) over the set

$$
\mathcal{K}_{3}=\left\{(\beta, Y)\left|\beta \in \mathbb{R}, Y \geq 0, \int_{\mathbb{R}}\right|\left(e^{y}-1\right) Y(x)-h(x) \mid \nu(d x)<\infty, I_{3}(\beta, Y)<\infty\right\}
$$

The solution to this optimisation problems can be only of the form:

$$
Y(y)=\left(1-\beta^{*}\left(e^{y}-1\right)\right)^{-1}
$$

with $\beta^{*}$ satisfying 45 .

Proof of Corollaries 1, 2, 3The proofs of Corollaries can be performed by using KunhTucker theorem to minimize the mentioned integrals under constraints (see [39])

\section{$3 \quad f$-divergence minimal EMM's for change-point model}

Here we describe all locally equivalent martingale measures (EMMs) for change point model leaving on our probability space equipped with initially enlarged filtration, and in particular in relation to the sets of EMMs of the two associated Levy models $L$ and $\tilde{L}$. We denote these sets by $\mathcal{M}(P)$ and $\mathcal{M}(\tilde{P})$ respectively. 


\subsection{EMMs for change-point model}

We assume that the sets $\mathcal{M}(P)$ and $\mathcal{M}(\tilde{P})$ are non-empty. Let $Q \in \mathcal{M}(P)$ and $\tilde{Q} \in \mathcal{M}(\tilde{P})$. We introduce the Radon-Nikodym density processes $\zeta=\left(\zeta_{t}\right)_{t \geq 0}$ and $\widetilde{\zeta}=\left(\tilde{\zeta}_{t}\right)_{t \geq 0}$ given by

$$
\zeta_{t}=\frac{d Q_{t}}{d P_{t}}, \quad \tilde{\zeta}_{t}=\frac{d \tilde{Q}_{t}}{d \tilde{P}_{t}}
$$

where $Q_{t}, P_{t}, \tilde{Q}_{t}, \tilde{P}_{t}$ stand for the restrictions of the corresponding measures to the $\sigma$ algebra $\mathcal{G}_{t}$.

We also introduce for all $t>0$

$$
v_{t}=\frac{d \tilde{P}_{t}}{d P_{t}}
$$

then

$$
V_{t}=\mathbf{1}_{\llbracket 0, \tau \rrbracket}(t)+\frac{v_{t}}{v_{\tau}} \mathbf{1}_{\rrbracket \tau,+\infty \llbracket}(t)
$$

We remark that the measure $\mathbb{P}$ which is the law of $X$ verify for $t \geq 0$ :

$$
\frac{d \mathbb{P}_{t}}{d P_{t}}=V_{t} .
$$

To describe all EMMs leaving on our space we define the process $z=\left(z_{t}\right)_{t \geq 0}$ given by

$$
z_{t}=\zeta_{t} \mathbf{1}_{\llbracket 0, \tau \rrbracket}(t)+\zeta_{\tau} \frac{\tilde{\zeta}_{t}}{\tilde{\zeta}_{\tau}} \mathbf{1}_{\rrbracket \tau,+\infty \llbracket}(t)
$$

Finally, we consider the measure $\mathbb{Q}$ such that

$$
\frac{d \mathbb{Q}_{t}}{d \mathbb{P}_{t}}=c(\tau) z_{t}
$$

where $c(\cdot)$ is a measurable function $[0, T] \rightarrow \mathbb{R}^{+, *}$ with $\mathbb{E} c(\tau)=1$.

Proposition 1. A measure $\mathbb{Q}$ is an equivalent martingale measure for the exponential model (4) related to the process $X$ iff its density process has the form (29).

Proof First we show that the process $Z=\left(Z_{t}\right)_{t \geq 0}$ given by

$$
Z_{t}=c(\tau) z_{t}
$$

is a density process with respect to $\mathbb{P}$ and that the process $S=\left(S_{t}\right)_{t \geq 0}$ such that

$$
S_{t}=e^{L_{t}} \mathbf{1}_{\llbracket 0, \tau \rrbracket}(t)+S_{\tau} e^{\tilde{L}_{t}-\tilde{L}_{\tau}} \mathbf{1}_{\rrbracket \tau,+\infty \llbracket}(t)
$$

is a $(\mathbb{Q}, \mathbb{F})$ - martingale.

We begin by noticing that if $M, \tilde{M}$ are two strictly positive $(\mathbb{P}, \mathbb{G})$ martingales on the same filtered probability space and $\tau$ is a stopping time independent of $M$ and $\tilde{M}$, then $N=\left(N_{t}\right)_{t \geq 0}$ such that

$$
N_{t}=c(\tau)\left[M_{t} \mathbf{1}_{\llbracket 0, \tau \rrbracket}(t)+M_{\tau} \frac{\tilde{M}_{t}}{\tilde{M}_{\tau}} \mathbf{1}_{\rrbracket \tau,+\infty \llbracket}(t)\right]
$$


is a $(\mathbb{P}, \mathbb{F})$ martingale. This fact, for example, can be proved by the conditioning with respect to $\mathcal{H}_{T}$ and use the facts that $M$ and $\tilde{M}$ are $(\mathbb{P}, \mathbb{G})$ martingales. To show that $Z$ is a $(\mathbb{P}, \mathbb{F})$-martingale, we prove an equivalent fact that $\left(V_{t} Z_{t}\right)_{t \geq 0}$ is a $(P, \mathbb{F})$ martingale. In fact, the relations (27), (28), (301) and previous remark with $M_{t}=\zeta_{t}$ and $\tilde{M}_{t}=\tilde{\zeta}_{t} v_{t}$ gives the result. Furthermore, taking conditional expectation with respect to $\mathcal{H}_{T}$ and using the fact that $\zeta$ and $\tilde{\zeta}$ are density processes independent from $\tau$, we see that $\mathbb{E} Z_{t}=1$. To show that $S=\left(S_{t}\right)_{t \geq 0}$ is $(\mathbb{Q}, \mathbb{F})$-martingale we establish that $\left(V_{t} Z_{t} S_{t}\right)_{t \geq 0}$ is a $(P, \mathbb{F})$ - martingale. For this we use the same remark with $M_{t}=e^{L_{t}} \zeta_{t}$ and $\tilde{M}_{t}=v_{t} \tilde{\zeta}_{t} e^{\tilde{L}_{t}}$.

Conversely, $Z$ is the density of any equivalent martingale measure if and only if $\left(Z_{t} S_{t}\right)_{t \geq 0}$ is a $(\mathbb{P}, \mathbb{F})$ - martingale. But the last fact is equivalent to: for any bounded stopping time $\sigma$,

$$
\mathbb{E}\left(Z_{\sigma} S_{\sigma}\right)=1
$$

Replacing $\sigma$ by $\sigma \wedge \tau$ in previous expression we deduce that $\left(Z_{t \wedge \tau}\right)_{t \geq 0}$ is the density of a martingale measure for $\left(e^{L_{t \wedge \tau}}\right)_{t \geq 0}$. In the same way, using the martingale properties of $Z$ we get for any bounded stopping time $\sigma$ that

$$
\mathbb{E}\left(\frac{Z_{\sigma} S_{\sigma}}{Z_{\sigma \wedge \tau} S_{\sigma \wedge \tau}}\right)=1
$$

and so $\left(\frac{Z_{t}}{Z_{t \wedge \tau}}\right)_{t \geq \tau}$ is the density of an equivalent martingale measure for $\left(e^{\tilde{L}_{t}-\tilde{L}_{t \wedge \tau}}\right)_{t \geq \tau}$.

\subsection{From EMM's to $f$-divergence minimal EMM's.}

In the following theorem we give an expression for the density of the $f$-divergence minimal EMM's $\mathbb{Q}_{T}^{*}$ with respect to $\mathbb{P}_{T}$ in our change-point framework. We set for $t \in[0, T]$

$$
z_{T}^{*}(t)=\zeta_{t}^{*} \frac{\tilde{\zeta}_{T}^{*}}{\tilde{\zeta}_{t}^{*}}
$$

where $\zeta^{*}$ and $\tilde{\zeta}^{*}$ are the densities of the $f$-divergence EMM's $Q^{*}$ and $\tilde{Q}^{*}$ with respect to $P$ and $\tilde{P}$ respectively.

We introduce the following hypotheses:

$\left(\mathcal{H}_{1}\right)$ : The $f$-divergence minimal equivalent martingale measures $Q^{*}$ and $\tilde{Q}^{*}$ relative to $L$ and $\tilde{L}$ exist.

$\left(\mathcal{H}_{2}\right)$ : The $f$-divergence minimal equivalent martingale measures $Q^{*}$ and $\tilde{Q}^{*}$ preserve the Levy property and are invariant under scaling.

$\left(\mathcal{H}_{3}\right)$ : For all $c>0$ and $t \in[0, T]$, we have

$$
\sup _{0 \leq t \leq T} \mathbb{E}\left|z_{T}^{*}(t) f^{\prime}\left(c z_{T}^{*}(t)\right)\right|<\infty
$$

where $\mathbb{E}$ is the expectation with respect to $\mathbb{P}$. 
Theorem 2. Assume that $f$ is a strictly convex function, $f \in C^{1}\left(\mathbb{R}^{+, *}\right)$, and that $\left(\mathcal{H}_{1}\right)$, $\left(\mathcal{H}_{2}\right),\left(\mathcal{H}_{3}\right)$ hold. If the f-divergence EMM's $\mathbb{Q}^{*}$ for the change-point model (4) exists, then

$$
\frac{d \mathbb{Q}_{T}^{*}}{d \mathbb{P}_{T}}=c(\tau) z_{T}^{*}(\tau)
$$

where $c(\cdot)$ is a measurable function $[0, T] \rightarrow \mathbb{R}^{+}$such that $\mathbb{E} c(\tau)=1$.

For $c>0$, let

$$
\lambda_{t}(c)=\mathbb{E}\left[z_{T}^{*}(t) f^{\prime}\left(c z_{T}^{*}(t)\right)\right]
$$

and let $c_{t}(\lambda)$ be its right-continuous inverse.

If in addition there exists $\lambda^{*}$ such that

$$
\int_{0}^{T} c_{t}\left(\lambda^{*}\right) d \alpha(t)=1
$$

then the $f$-minimal equivalent martingale measure for a change-point model exists and the density $Z_{T}^{*}(\tau)$ of $\mathbb{Q}_{T}^{*}$ with respect to $\mathbb{P}_{T}$ is equal to

$$
Z_{T}^{*}(\tau)=c^{*}(\tau) z_{T}^{*}(\tau)
$$

where $c^{*}(t)=c_{t}\left(\lambda^{*}\right)$ for $t \in[0, T]$.

Corollary 4. Assume that $f$ is power function, $f(x)=c_{\gamma} x^{\gamma+2}$. Then under $\left(\mathcal{H}_{1}\right)$ the $f$-divergence EMM for change-point model exist and $Z_{T}^{*}(\tau)=c^{*}(\tau) z_{T}^{*}(\tau)$ with

$$
c^{*}(t)=\frac{\left[\mathbb{E}\left(z_{T}^{*}(t)^{\gamma+2}\right)\right]^{-\frac{1}{\gamma+1}}}{\int_{0}^{T}\left[\mathbb{E}\left(z_{T}^{*}(t)^{\gamma+2}\right)\right]^{-\frac{1}{\gamma+1}} d \alpha(t)}
$$

Corollary 5. Assume that $f(x)=x \ln (x)$. Then under $\left(\mathcal{H}_{1}\right)$ the f-divergence EMM for change-point model exist and $Z_{T}^{*}(\tau)=c^{*}(\tau) z_{T}^{*}(\tau)$ with

$$
c^{*}(t)=\frac{e^{-\mathbb{E}\left(z_{T}^{*}(t) \ln z_{T}^{*}(t)\right)}}{\int_{0}^{T} e^{-\mathbb{E}\left(z_{T}^{*}(t) \ln z_{T}^{*}(t)\right)} d \alpha(t)} .
$$

Corollary 6. Assume that $f(x)=-\ln (x)$. Then under $\left(\mathcal{H}_{1}\right)$ the $f$-divergence EMM for change-point model exist and $Z_{T}^{*}(\tau)=c^{*}(\tau) z_{T}^{*}(\tau)$ with $c^{*}(t)=1$.

Remark 1. We can also express the factor $c^{*}(t)$ in terms of $f$-divergences of the processes $L$ and $\tilde{L}$. Namely, one can see easily that

$$
\mathbb{E}\left(z_{T}^{*}(t)^{\gamma+2}\right)=\mathbb{E}\left(\zeta_{t}^{* \gamma+2}\right) \mathbb{E}\left(\tilde{\zeta}^{*}{ }_{T-t}^{\gamma+2}\right)
$$

and that

$$
\begin{gathered}
\mathbb{E}\left(z_{T}^{*}(t) \ln z_{T}^{*}(t)\right)=\mathbb{E}\left(\zeta_{t}^{*} \ln \zeta_{t}^{*}\right)+\mathbb{E}\left(\tilde{\zeta}_{T-t}^{*} \ln \tilde{\zeta}_{T-t}^{*}\right), \\
\mathbb{E}\left(-\ln z_{T}^{*}(t)\right)=\mathbb{E}\left(-\ln \zeta_{t}^{*}\right)+\mathbb{E}\left(-\ln \tilde{\zeta}_{T-t}^{*}\right) .
\end{gathered}
$$

In turn, the last quantities can be easily expressed via the corresponding Girsanov parameters using Ito formula as it was done in Lemma 2. 
Proof of Theorem 2 Since $\mathbb{Q}^{*}$ is an equivalent martingale measure we have from (29) that

$$
f\left(\mathbb{Q}_{T}^{*} \mid \mathbb{P}_{T}\right)=\mathbb{E}\left[f\left(c(\tau) \zeta_{\tau} \frac{\tilde{\zeta}_{T}}{\tilde{\zeta}_{\tau}}\right)\right]
$$

It follows from the independence of $L, \tilde{L}$ and $\tau$ that

$$
\mathbb{E}\left[f\left(c(\tau) \zeta_{\tau} \frac{\tilde{\zeta}_{T}}{\tilde{\zeta}_{\tau}}\right) \mid \tau=t\right]=\mathbb{E}\left[f\left(c(t) \zeta_{t} \frac{\tilde{\zeta}_{T}}{\tilde{\zeta}_{t}}\right)\right]
$$

Now, the independence of $L$ and $\tilde{L}$ implies the conditional independence of $\zeta$ and $\tilde{\zeta}$ given $\sigma\left(L_{s}, s \leq T\right)$. Using the invariance of $f$ under scaling, we see that in order to minimize $f$-divergence, the measure $\mathbb{Q}$ should be such that $\zeta$ is the density of an $f$-divergence minimal martingale measure for $\left(e^{L_{t}}\right)_{t \geq 0}$ and $\tilde{\zeta}$ the density of an $f$-divergence minimal martingale measure for $\left(e^{\tilde{L}_{t}}\right)_{t \geq 0}$. Hence (32) holds.

To find $f$-divergence minimal equivalent martingale measure we have to minimize the function

$$
F(c)=\int_{0}^{T} \mathbb{E}\left[f\left(c(t) z_{T}^{*}(t)\right)\right] d \alpha(t)
$$

over all cadlag functions $c:[0, T] \rightarrow \mathbb{R}^{+, *}$ such that $\mathbb{E} c(\tau)=1$. For that we consider the linear space $\mathcal{L}$ of such cadlag functions $c:[0 ; T] \rightarrow \mathbb{R}$ with the norm $\|c\|=$ $\sup _{t \in[0, T]}|c(t)|$ and also the cone of such positive functions.

We apply Kuhn-Tucker theorem (see [39]) to the function

$$
F_{\lambda}(c)=F(c)-\lambda \int_{0}^{T}(c(t)-1) d \alpha(t)
$$

with Lagrangian factor $\lambda>0$. We show that the Frechet derivative $\frac{\partial F_{\lambda}}{\partial c}$ of $F_{\lambda}(c)$, defined by

$$
\lim _{\|\delta\| \rightarrow 0} \frac{\left|F_{\lambda}(c+\delta)-F_{\lambda}(c)-\frac{\partial F_{\lambda}}{\partial c} \delta\right|}{\|\delta\|}=0
$$

is equal to:

$$
\frac{\partial F_{\lambda}}{\partial c}(\delta)=\int_{0}^{T}\left(\mathbb{E}\left[f^{\prime}\left(c(t) z_{T}^{*}(t)\right) z_{T}^{*}(t)\right]-\lambda\right) \delta(t) d \alpha(t)
$$

In fact, by the Taylor formula, we have for $\delta \in \mathcal{L}$ :

$$
\begin{gathered}
F_{\lambda}(c+\delta)-F_{\lambda}(c)-\frac{\partial F_{\lambda}}{\partial c} \delta= \\
\int_{0}^{T} \mathbb{E}\left[\left(f^{\prime}\left((c(t)+\theta(t)) z_{T}^{*}(t)\right)-f^{\prime}\left(c(t) z_{T}^{*}(t)\right)\right) z_{T}^{*}(t)\right] \delta(t) d \alpha(t)
\end{gathered}
$$

where $\theta(t)$ is a function which takes values in the interval $[0, \delta(t)]$. We remark that the modulus of the right-hand side in the previous equality is bounded from above by:

$$
A_{T}=\sup _{t \in[0, T]} \mathbb{E}\left[\left|f^{\prime}\left((c(t)+\theta(t)) z_{T}^{*}(t)\right)-f^{\prime}\left(c(t) z_{T}^{*}(t)\right)\right| z_{T}^{*}(t)\right]\|\delta\|
$$


Since $f^{\prime}$ is continuous and increasing and the functions $c$ and $\delta$ are bounded, hypothesis $\left(\mathcal{H}_{3}\right)$ implies that $A_{T}$ is finite. We conclude by Lebesgue's dominated convergence theorem that (34) holds and then (35).

Then, in order to $\frac{\partial F_{\lambda}}{\partial c} \delta=0$ for all $\delta \in \mathcal{L}$, it is necessary and sufficient to take $c$ such that

$$
\mathbb{E}\left[z_{T}^{*}(t) f^{\prime}\left(c(t) z_{T}^{*}(t)\right)\right]-\lambda=0 \quad \alpha \text {-a.s. }
$$

Finally, for each $c>0$ and $t \in[0, T]$ we consider the function

$$
\lambda_{t}(c)=\mathbb{E}\left[z_{T}^{*}(t) f^{\prime}\left(c z_{T}^{*}(t)\right)\right] .
$$

We see easily that it is increasing in $c$ and that its right-continuous inverse $c_{t}(\lambda)$ satisfies:

$$
\lambda=\mathbb{E}\left[z_{T}^{*}(t) f^{\prime}\left(c_{t}(\lambda) z_{T}^{*}(t)\right)\right]
$$

Now, to obtain a minimizer $c^{*}$, it remains to find, if it exists, $\lambda^{*}$ which satisfies (33).

Proof of Corollaries 4, 5 and 6. First of all we remark that for common $f$-divergences, the hypothesis $\left(\mathcal{H}_{1}\right)$ implies $\left(\mathcal{H}_{2}\right)$ and $\left(\mathcal{H}_{3}\right)$. Then, we obtain in power case $f(x)=$ $c_{\gamma} x^{\gamma+2}$ that $\lambda_{t}(c)=(\gamma+2) c_{\gamma} c^{\gamma+1} \mathbb{E}\left[z_{T}^{*}(t)^{\gamma+2}\right]$. For $f(x)=x \ln (x)$ we get $\lambda_{t}(c)=$ $\mathbb{E}\left[z_{T}^{*}(t) \ln z_{T}^{*}(t)\right]+\ln c+1$. In the case $f(x)=-\ln x$ we get $\lambda_{t}(c)=-1 / c$. Finally, we write down $c_{t}(\lambda)$ and we integrate with respect to $\alpha$ to find $\lambda^{*}$ and the expression of $c^{*}(t)$.

Example: A change-point Black-Scholes model. We apply the previous results when $L$ and $\tilde{L}$ define Black-Scholes type models. Therefore, we assume that $L$ and $\tilde{L}$ are continuous Levy processes with characteristics $(b, c, 0)$ and $(\tilde{b}, c, 0)$ respectively, $c>$ 0 . As is well known, the initial models will be complete, with a unique equivalent martingale measure which defines a unique price for options. However, in our changepoint model the martingale measure is not unique, and we have an infinite set of martingale measures of the form

$$
\frac{d \mathbb{Q}_{T}}{d \mathbb{P}_{T}}(X)=c(\tau) \exp \left(\int_{0}^{T} \beta_{s} d X_{s}^{c}-\frac{1}{2} \int_{0}^{T} \beta_{s}^{2} c d s\right)
$$

where $c(\cdot)$ is a measurable function $[0, T] \rightarrow \mathbb{R}^{+, *}$ such that $\mathbb{E}[c(\tau)]=1$ and

$$
\beta_{s}=-\frac{1}{c}\left[\left(b+\frac{c}{2}\right) \mathbf{1}_{\llbracket 0, \tau \rrbracket}(s)+\left(\tilde{b}+\frac{c}{2}\right) \mathbf{1}_{\rrbracket \tau,+\infty \llbracket}(s)\right]
$$

If for example $f(x)=c_{\gamma} x^{\gamma+2}$ with $\gamma \neq-1,-2$ then applying Theorem 2 , we get

$$
c^{*}(t)=\frac{e^{-\frac{\gamma+2}{2 c}\left[\left(b+\frac{c}{2}\right)^{2} t+\left(\tilde{b}+\frac{c}{2}\right)^{2}(T-t)\right]}}{\int_{0}^{T} e^{-\frac{\gamma+2}{2 c}\left[\left(b+\frac{c}{2}\right)^{2} t+\left(\tilde{b}+\frac{c}{2}\right)^{2}(T-t)\right]} d \alpha(t)}
$$

If $f(x)=x \ln (x)$, then

$$
c^{*}(t)=\frac{e^{-\frac{1}{2 c}\left[\left(b+\frac{c}{2}\right)^{2} t+\left(\tilde{b}+\frac{c}{2}\right)^{2}(T-t)\right]}}{\int_{0}^{T} e^{-\frac{1}{2 c}\left[\left(b+\frac{c}{2}\right)^{2} t+\left(\tilde{b}+\frac{c}{2}\right)^{2}(T-t)\right]} d \alpha(t)}
$$

If $f(x)=-\ln (x)$, then $c^{*}(t)=1$. 


\section{Optimal strategies for utility maximization}

We start by recalling some useful basic facts about optimal strategies for utility maximization. Then some decomposition formulas will be given which permit us to find optimal strategies. We end up by giving the formulas for optimal strategies for utility maximization in change-point setting for both initially and progressively enlarged filtrations.

\subsection{Some known facts}

In this subsection, we are interested in finding optimal strategies for terminal wealth with respect to some utility functions. More precisely, we assume that our financial market consists of two assets : a non-risky asset $B$, with interest rate $r$, and a risky asset $S$, modelled using the change-point Levy model defined in (4). We denote by $\vec{S}=(B, S)$ the price process and by $\vec{\Phi}=\left(\phi^{0}, \phi\right)$ the amount of money invested in each asset. According to usual terminology, a predictable $\vec{S}$-integrable process $\vec{\Phi}$ is said to be a self-financing admissible strategy if for every $t \in[0, T]$ and $x$ initial capital

$$
\vec{\Phi}_{t} \cdot \vec{S}_{t}=x+\int_{0}^{t} \vec{\Phi}_{u} \cdot d \vec{S}_{u}
$$

where the stochastic integral in the right-hand side is bounded from below. Here denotes the scalar product. We will denote by $\mathcal{A}$ the set of all self-financing admissible strategies. In order to avoid unnecessary complications, we will assume again that the interest rate $r$ is 0 , so that starting with an initial capital $x$, terminal wealth at time $T$ is

$$
V_{T}(\phi)=x+\int_{0}^{T} \phi_{s} d S_{s}
$$

Let $u$ denote a strictly increasing, strictly concave, continuously differentiable function on $\operatorname{dom}(u)=\{x \in \mathbb{R} \mid u(x)>-\infty\}$ which satisfies

$$
\begin{gathered}
u^{\prime}(+\infty)=\lim _{x \rightarrow+\infty} u^{\prime}(x)=0, \\
u^{\prime}(\underline{x})=\lim _{x \rightarrow \underline{x}} u^{\prime}(x)=+\infty
\end{gathered}
$$

where $\underline{x}=\inf \{u \in \operatorname{dom}(u)\}$.

We will say that $\phi^{*}$ defines an optimal strategy with respect to $u$ if

$$
\mathbb{E}_{P}\left[u\left(x+\int_{0}^{T} \phi_{s}^{*} d S_{s}\right)\right]=\sup _{\phi \in \mathcal{A}} \mathbb{E}_{P}\left[u\left(x+\int_{0}^{T} \phi_{s} d S_{s}\right)\right]
$$

As in [35], we will say that $\phi^{*}$ is an asymptotically optimal strategy if there exists a sequence of admissible strategies $\left(\phi^{(n)}\right)_{n \geq 1}$ such that

$$
\lim _{n \rightarrow+\infty} E\left[u\left(x+\int_{0}^{T} \phi_{s}^{(n)} d S_{s}\right)\right]=\sup _{\phi \in \mathcal{A}} E\left[u\left(x+\int_{0}^{T} \phi_{s} d S_{s}\right)\right]
$$


As known, there is a strong link between this optimization problem and the previous problem of finding $f$-divergence minimal martingale measures. Let $f$ be the convex conjugate function of $u$ :

$$
f(y)=\sup _{x \in \mathbb{R}}\{u(x)-x y\}=u(I(y))-y I(y)
$$

where $I=\left(u^{\prime}\right)^{-1}=-f^{\prime}$. We recall that in particular

$$
\begin{aligned}
& \text { if } u(x)=\ln (x) \text { then } f(x)=-\ln (x)-1, \\
& \text { if } u(x)=\frac{x^{p}}{p}, p<1 \text { then } f(x)=-\frac{p-1}{p} x^{\frac{p}{p-1}}, \\
& \text { if } u(x)=1-e^{-x} \text { then } f(x)=1-x+x \ln (x) .
\end{aligned}
$$

The following result gives us the relation between portfolio optimization and f-minimal martingale measures.

Theorem 3. (cf. [27]) Let $x \in \mathbb{R}^{+}$be fixed and $f \in C^{1}\left(\mathbb{R}^{+, *}\right)$. Let $Q^{*}$ be an equivalent martingale measure which satisfies

$$
\mathbb{E}_{P}\left|f\left(\lambda \frac{d Q_{T}^{*}}{d P_{T}}\right)\right|<\infty, \quad \mathbb{E}_{Q^{*}}\left|f^{\prime}\left(\lambda \frac{d Q_{T}^{*}}{d P_{T}}\right)\right|<\infty
$$

for $\lambda$ such that

$$
-\mathbb{E}_{Q^{*}} f^{\prime}\left(\lambda \frac{d Q_{T}^{*}}{d P_{T}}\right)=x
$$

Then, if $Q^{*}$ is an $f$-divergence minimal martingale measure, there exists a predictable function $\phi^{*}$ such that $\left(\int_{0}^{*} \phi_{u}^{*} d S_{u}\right)$ is a $Q^{*}$-martingale and

$$
-f^{\prime}\left(\lambda \frac{d Q_{T}^{*}}{d P_{T}}\right)=x+\int_{0}^{T} \phi_{u}^{*} d S_{u}
$$

If the last relation holds, then $\vec{\Phi}=\left(\phi^{0}, \phi\right)$ with $\phi_{t}^{0}=x+\int_{0}^{t} \phi_{u} d S_{u}-\phi_{t} S_{t}$ is an asymptotically optimal portfolio strategy. Moreover, if $\underline{x}>-\infty$, this strategy is optimal.

Proof The first part of the Theorem is a slight adaptation of [35]. We do however recall the proof for the reader's ease. We denote $Z_{T}=\frac{d Q_{T}^{*}}{d P_{T}}$.

As $f^{\prime}$ is strictly increasing, continuous and due to imposed integrability conditions, the function $\lambda \mapsto E_{Q^{*}}\left[f^{\prime}\left(\lambda Z_{T}\right)\right]$ is also strictly increasing and continuous. Furthermore, since $f^{\prime}=-\left(u^{\prime}\right)^{-1}$, we have $\lim _{\lambda \rightarrow 0} E_{Q^{*}}\left[f^{\prime}\left(\lambda Z_{T}\right)\right]=-\infty$ and $\lim _{\lambda \rightarrow+\infty} E_{Q^{*}}\left[f^{\prime}\left(\lambda Z_{T}\right)\right]=$ $-\underline{x}$. Hence, for all $x>\underline{x}$, there exists a unique $\lambda>0$ such that $E_{Q^{*}}\left[f^{\prime}\left(\lambda Z_{T}\right)\right]=-x$. As $Q^{*}$ is minimal for the function $x \mapsto f(\lambda x)$, it follows from Theorem 3.1 of [27], that there exists a predictable process $\phi^{*}$ such that

$$
-f^{\prime}\left(\lambda Z_{T}\right)=x+\left(\phi^{*} \cdot S\right)_{T}
$$

and furthermore $\phi^{*} \cdot S$ defines a $Q^{*}$-martingale. Then, from the definition of the convex conjugate, we have

$$
u\left(x+\left(\phi^{*} \cdot S\right)_{T}\right)=f\left(\lambda Z_{T}\right)-\lambda Z_{T} f^{\prime}\left(\lambda Z_{T}\right)
$$


and, hence,

$$
E_{P}\left[\left|u\left(x+\left(\phi^{*} \cdot S\right)_{T} \mid\right] \leq E_{P}\right| f\left(\lambda Z_{T}\right) \mid+\lambda E_{P}\left[Z_{T}\left|f^{\prime}\left(\lambda Z_{T}\right)\right|\right]<\infty .\right.
$$

If now $\phi$ denotes any admissible strategy, we have from $u(x) \leq f(y)+x y$ for all $x, y \in \mathbb{R}^{+, *}$ that

$$
\begin{aligned}
u\left(x+(\phi \cdot S)_{T}\right) & \leq\left(x+(\phi \cdot S)_{T}\right) \lambda Z_{T}+f\left(\lambda Z_{T}\right) \\
& \leq\left(x+(\phi \cdot S)_{T}\right) \lambda Z_{T}+u\left(x+\left(\phi^{*} \cdot S\right)_{T}\right)+\lambda Z_{T} f^{\prime}\left(\lambda Z_{T}\right)
\end{aligned}
$$

Taking expectation, we obtain since $E_{P}\left(Z_{T} f^{\prime}\left(\lambda Z_{T}\right)\right)=-x$, that

$$
E_{P}\left[u\left(x+(\phi \cdot S)_{T}\right)\right] \leq E_{P}\left[u\left(x+\left(\phi^{*} \cdot S\right)_{T}\right]+\lambda E_{Q^{*}}\left[(\phi \cdot S)_{T}\right]\right.
$$

Now, under $Q^{*},(\phi \cdot S)$ is a local martingale, so that $E_{Q^{*}}\left[(\phi \cdot S)_{T}\right] \leq 0$. Therefore,

$$
E_{P}\left[u\left(x+(\phi \cdot S)_{T}\right)\right] \leq E_{P}\left[u\left(x+\left(\phi^{*} \cdot S\right)_{T}\right)\right]
$$

Furthermore, if $\underline{x}>-\infty$, we note that $\left(\phi^{*} \cdot S\right)_{T} \geq \underline{x}-x$, so that $\phi^{*}$ defines an admissible strategy, and hence is a $u$-optimal strategy.

When $\underline{x}=-\infty$, we can construct using the definition of $\mathcal{A}$ a sequence of admissible strategies $\phi^{(n)}$ such that for all $0 \leq t \leq T,\left(\phi^{(n)} \cdot S\right)_{t} \geq-n$ and such that

$$
\lim _{n \rightarrow+\infty} E\left[u\left(x+\left(\phi^{(n)} \cdot S\right)_{T}\right)\right]=\sup _{\phi \in \mathcal{A}} E\left[u\left(x+(\phi \cdot S)_{T}\right)\right] .
$$

Finally, $\phi^{*}$ is asymptotically $u$-optimal.

In the following theorem proved in [6] we give a unified expression of u-optimal strategy for exponential Levy model. We denote by $\left(\beta^{*}, Y^{*}\right)$ the Girsanov parameters for changing of the measure $P$ into $Q^{*}$. We put also

$$
\xi_{t}(x)=E_{Q^{*}}\left[f^{\prime}\left(x Z_{T-t}\right) Z_{T-t}\right]
$$

Theorem 4. Let $u$ be a $\mathcal{C}^{3}(] \underline{x},+\infty[)$ utility function and $f$ its convex conjugate. Assume there exists an $f$-minimal martingale measure $Q^{*}$ which preserves the Lévy property and such that the integrability conditions are satisfied: for all $\lambda>0$ and all compact set $K \subseteq \mathbb{R}^{+}$

$$
E_{P}\left|f\left(\lambda Z_{T}\right)\right|<+\infty, \quad E_{Q}\left|f^{\prime}\left(\lambda Z_{T}\right)\right|<+\infty, \quad \sup _{t \leq T} \sup _{\lambda \in K} E_{Q}\left[f^{\prime \prime}\left(\lambda Z_{t}\right) Z_{t}\right]<+\infty .
$$

Then for any fixed initial capital $x>\underline{x}$, there exists an asymptotically u-optimal strategy $\phi^{*}$. In addition, $\phi^{*}$ defines a u-optimal strategy as soon as $\underline{x}>-\infty$.

Furthermore, if $c \neq 0$, we have

$$
\phi_{s}^{*}=-\frac{\lambda \beta^{*} Z_{s-}}{S_{s-}} \xi_{s}\left(\lambda Z_{s-}\right)
$$


where $\lambda$ is a unique solution to the equation $E_{Q^{*}}\left(-f^{\prime}\left(\lambda Z_{T}\right)\right)=x$.

If $c=0$, supp $(\nu) \neq \emptyset$ and it contains zero, and $Y^{*}$ is not identically 1 , then $f^{\prime \prime}(x)=$ $a x^{\gamma}$ with $a>0$ and $\gamma \in \mathbb{R}$, and

$$
\phi_{s}^{*}=-\frac{\lambda \alpha^{*} Z_{s-}}{S_{s-}} \xi_{s}\left(\lambda Z_{s-}\right)
$$

where again $\lambda$ is a unique solution to the equation $E_{Q^{*}}\left(-f^{\prime}\left(\lambda Z_{T}\right)\right)=x$ and the constant $\alpha^{*}$ is related with the second Girsanov parameter $Y^{*}$ by the formula:

$$
\alpha^{*}=\exp \left(-y_{0}\right) Y\left(y_{0}\right)^{\gamma} Y^{\prime}\left(y_{0}\right)
$$

where $y_{0}$ is chosen arbitrarily in supp $(\nu)$.

In the case of classical utilities we obtain the following result.

Proposition 2. Consider a Lévy process $X$ with characteristics $(b, c, \nu)$ and let $f$ be a function such that $f^{\prime \prime}(x)=a x^{\gamma}$, where $a>0$ and $\gamma \in \mathbb{R}$. Let $u_{f}$ be its concave conjugate. Assume there exist $\alpha, \beta \in \mathbb{R}$ and a measurable function $Y: \mathbb{R} \backslash\{0\} \rightarrow \mathbb{R}^{+}$ such that

$$
Y(y)=\left(f^{\prime}\right)^{-1}\left(f^{\prime}(1)+\alpha\left(e^{y}-1\right)\right)
$$

and such that the following properties hold:

$$
\begin{gathered}
Y(y)>0 \nu-a . e ., \\
\int_{|y| \geq 1}\left(e^{y}-1\right) Y(y) \nu(d y)<\infty . \\
b+\frac{1}{2} c+c \beta+\int_{\mathbb{R}}\left(\left(e^{y}-1\right) Y(y)-h(y)\right) \nu(d y)=0 .
\end{gathered}
$$

Then if $c \neq 0$, there exists an asymptotically optimal strategy $\phi^{*}$ given by

$$
\phi_{s}^{*}=\alpha_{\gamma}(x) \frac{\beta Z_{s-}^{\gamma+1}}{E_{Q^{*}}\left[Z_{s}^{\gamma+1}\right] S_{s-}},
$$

where

$$
\alpha_{\gamma}(x)=a-(\gamma+1)\left(x+f^{\prime}(1)\right) .
$$

If $c=0$ and $\operatorname{sipp}(\nu) \neq \emptyset$, then

$$
\phi_{s}^{*}=\alpha_{\gamma}(x) \frac{\alpha Z_{s-}^{\gamma+1}}{E_{Q^{*}}\left[Z_{s}^{\gamma+1}\right] S_{s-}},
$$

In addition, $\phi^{*}$ is optimal as soon as $\gamma \neq-1$. 


\subsection{A decomposition formula for initially enlarged filtration}

We use the structure of $\mathbb{Q}^{*}$ presented in Theorem 2 to write down a decomposition formula mentioned in Theorem 3 for $f^{\prime}\left(\lambda Z_{T}^{*}(\tau)\right)$. First of all we give the expressions for Girsanov parameters when changing the measure $\mathbb{P}$ into $\mathbb{Q}^{*}$.

Lemma 4. Let Girsanov parameters of the $f$-divergence minimal equivalent martingale measures $Q^{*}$ and $\tilde{Q}^{*}$ are $\left(\beta^{*}, Y^{*}\right)$ and $\left(\tilde{\beta}^{*}, \tilde{Y}^{*}\right)$ respectively. Then the Girsanov parameters when changing from $\mathbb{P}$ to $\mathbb{Q}^{*}$ are:

$$
\begin{gathered}
\beta_{t}^{*}(\tau)=\beta^{*} \mathbf{1}_{\llbracket 0, \tau \rrbracket}(t)+\tilde{\beta}^{*} \mathbf{1}_{\rrbracket \tau,+\infty \llbracket}(t) \\
Y_{t}^{*}(\tau)=Y^{*} \mathbf{1}_{\llbracket 0, \tau \rrbracket}(t)+\tilde{Y}^{*} \mathbf{1}_{\rrbracket \tau,+\infty \llbracket}(t) .
\end{gathered}
$$

Next, we introduce for fixed $u \in[0, T], x \geq 0$ and $t \in[0, T]$ the quantities

$$
\rho^{(u)}(t, x)=\mathbb{E}_{\mathbb{Q}^{*}}\left(f^{\prime}\left(Z_{T}^{*}(\tau)\right) \mid \tau=u, Z_{t}^{*}(u)=x\right)
$$

and we remark that

$$
\rho^{(u)}(t, x)=\mathbb{E}_{\mathbb{Q}_{u}^{*}}\left(f^{\prime}\left(Z_{T}^{*}(u)\right) \mid Z_{t}^{*}(u)=x\right)
$$

where $\mathbb{Q}_{u}^{*}$ is conditional probability $\mathbb{Q}^{*}$ given $\tau=u$. We notice that for regular conditional probabilities and for right-continuous versions of conditional expectations we have: $\mathbb{P}$-a.s. for all $t \in[0, T]$

$$
\mathbb{E}_{\mathbb{Q}^{*}}\left(f^{\prime}\left(Z_{T}^{*}(\tau)\right) \mid \mathcal{F}_{t}\right)=\rho^{(\tau)}\left(t, Z_{t}^{*}(\tau)\right)
$$

To simplify the notation we introduce $\eta_{T-t}(u)$ such that

$$
\eta_{T-t}(u)=\frac{z_{T}^{*}(u)}{z_{t}^{*}(u)}
$$

As a consequence of previous formulas, we have

$$
\rho^{(u)}(t, x)=\mathbb{E}\left[\eta_{T-t}(u) f^{\prime}\left(x \eta_{T-t}(u)\right)\right]
$$

Now, we would like to use Ito formula for $\rho^{(u)}\left(t, Z_{t}^{*}(u)\right)$. But the mentioned function is not sufficiently smooth and we will proceed by approximations. For that we construct a sequence of functions $\left(\phi_{n}\right)_{n \geq 1}$.

Lemma 5. Let $f$ be convex function belonging to $C^{3}\left(\mathbb{R}^{+, *}\right)$. There exists a sequence of bounded functions $\left(\phi_{n}\right)_{n \geq 1}$, which are of class $\mathcal{C}^{2}$ on $\mathbb{R}^{+, *}$, increasing, such that for all $n \geq 1, \phi_{n}$ coincides with $f^{\prime}$ on the compact set $\left[\frac{1}{n}, n\right]$ and such that for sufficiently big $n$ the following inequalities hold for all $x, y>0$ :

$$
\left|\phi_{n}(x)\right| \leq 4\left|f^{\prime}(x)\right|+\alpha,\left|\phi_{n}^{\prime}(x)\right| \leq 3 f^{\prime \prime}(x),\left|\phi_{n}(x)-\phi_{n}(y)\right| \leq 5\left|f^{\prime}(x)-f^{\prime}(y)\right|
$$

where $\alpha$ is a real positive constant. 
Proof We set, for $n \geq 1$,

$$
\begin{gathered}
A_{n}(x)=f^{\prime}\left(\frac{1}{n}\right)-\int_{x \vee \frac{1}{2 n}}^{\frac{1}{n}} f^{\prime \prime}(y)(2 n y-1)^{2}(5-4 n y) d y \\
B_{n}(x)=f^{\prime}(n)+\int_{n}^{x \wedge(n+1)} f^{\prime \prime}(y)(n+1-y)^{2}(1+2 y-2 n) d y
\end{gathered}
$$

and finally

$$
\phi_{n}(x)=\left\{\begin{array}{l}
A_{n}(x) \text { if } 0 \leq x<\frac{1}{n} \\
f^{\prime}(x) \text { if } \frac{1}{n} \leq x \leq n, \\
B_{n}(x) \text { if } x>n
\end{array}\right.
$$

Proof. We can verify easily that $\phi_{n}$ coincide with $f^{\prime}$ on $\left[\frac{1}{n}, n\right]$ and that the properties (48) hold.

Now we replace $f^{\prime}$ by $\phi_{n}$ in previous formulas and we introduce

$$
\rho_{n}^{(u)}(t, x)=\mathbb{E}_{\mathbb{Q}^{*}}\left(\phi_{n}\left(Z_{T}^{*}(\tau)\right) \mid \tau=u, Z_{t}^{*}(u)=x\right)
$$

It is not difficult to see that

$$
\rho_{n}^{(u)}\left(t, Z_{T}^{*}(u)\right)=\mathbb{E}\left[\eta_{T-t}(u) \phi_{n}\left(x \eta_{T-t}(u)\right)\right]
$$

In the next lemma we give a decomposition formula for $\rho_{n}^{(u)}$. For that we put

$$
\xi_{t}^{(n, u)}(x)=\mathbb{E}\left[\eta_{T-t}^{2}(u) \phi_{n}^{\prime}\left(x \eta_{T-t}(u)\right)\right]
$$

and

$$
H_{t}^{(n, u)}(x, y)=\mathbb{E}\left(\eta_{T-t}(u)\left[\phi_{n}\left(x \eta_{T-t}(u) Y_{t}^{*}(y)\right)-\phi_{n}\left(x \eta_{T-t}(u)\right)\right]\right)
$$

Lemma 6. We have $\mathbb{Q}_{u}^{*}$-a.s., for all $t \leq T$,

$$
\begin{gathered}
E_{\mathbb{Q}_{u}^{*}}\left[\phi_{n}\left(Z_{T}^{*}(u)\right) \mid \mathcal{G}_{t}\right]=E_{\mathbb{Q}_{u}^{*}}\left[\phi_{n}\left(Z_{T}^{*}(u)\right)\right]+ \\
\int_{0}^{t} \beta_{s}^{*}(u) Z_{s-}^{*}(u) \xi_{s}^{(n, u)}\left(Z_{s-}^{*}(u)\right) d X_{s}^{(c), \mathbb{Q}_{u}^{*}}+\int_{0}^{t} \int_{\mathbb{R}} H_{s}^{(n, u)}\left(Z_{s-}^{*}(u), y\right)\left(\mu^{X}-\nu^{X, \mathbb{Q}_{u}^{*}}\right)(d s, d y)
\end{gathered}
$$

where $\nu^{X, \mathbb{Q}_{u}^{*}}$ is a compensator of the jump measure $\mu^{X}$ with respect to $\left(\mathbb{G}, \mathbb{Q}_{u}^{*}\right)$.

Proof In order to apply the Ito formula to $\rho_{n}^{(u)}$, we show that $\rho_{n}$ is twice continuously differentiable with respect to $x$ and once with respect to $t$ on the set $x \geq \epsilon, \epsilon>0$ and $t \in[0, T]$ and that the corresponding derivatives are bounded on the mentioned set. Then we apply the Ito formula to $\rho_{n}^{(u)}$ but stopped at stopping times

$$
s_{m}=\inf \left\{t \geq 0 \mid Z_{t}^{*}(u) \leq \frac{1}{m}\right\}
$$

with $m \geq 1$ and $\inf \{\emptyset\}=\infty$. 
From strong Markov property of Levy processes we have:

$$
\rho_{n}^{(u)}\left(t \wedge s_{m}, Z_{t \wedge s_{m}}^{*}(u)\right)=E_{\mathbb{Q}_{u}^{*}}\left(\phi_{n}\left(Z_{T}^{*}(u)\right) \mid \mathcal{G}_{t \wedge s_{m}}\right)
$$

and we remark that $\left(E_{\mathbb{Q}_{u}^{*}}\left(\phi_{n}\left(Z_{T}^{*}(u)\right) \mid \mathcal{G}_{t \wedge s_{m}}\right)\right)_{t \geq 0}$ is a $\mathbb{Q}_{u}^{*}$ - martingale. By Ito formula we obtain that:

$$
\begin{aligned}
& \rho_{n}^{(u)}(t \wedge\left.s_{m}, Z_{t \wedge s_{m}}^{*}(u)\right)=\rho_{n}^{(u)}\left(0, Z_{0}^{*}(u)\right)+\int_{0}^{t \wedge s_{m}} \frac{\partial \rho_{n}^{(u)}}{\partial s}\left(s, Z_{s-}^{*}(u)\right) d s+ \\
& \int_{0}^{t \wedge s_{m}} \frac{\partial \rho_{n}^{(u)}}{\partial x}\left(s, Z_{s-}^{*}(u)\right) d Z_{s-}^{*}(u)+\frac{1}{2} \int_{0}^{t \wedge s_{m}} \frac{\partial^{2} \rho_{n}^{(u)}}{\partial x^{2}}\left(s, Z_{s-}^{*}(u)\right) d\left\langle Z^{*, c}(u)\right\rangle_{s}+ \\
& \int_{0}^{t \wedge s_{m}} \int_{\mathbb{R}}\left[\rho_{n}^{(u)}\left(s, Z_{s-}^{*}(u)+y\right)-\rho_{n}^{(u)}\left(s, Z_{s-}^{*}(u)\right)-\frac{\partial \rho_{n}^{(u)}}{\partial x}\left(s, Z_{s-}^{*}(u)\right) y\right] \mu^{Z^{*}}(d s, d y)
\end{aligned}
$$

Then we can write that

$$
\rho_{n}^{(u)}\left(t \wedge s_{m}, Z_{t \wedge s_{m}}^{*}(u)\right)=A_{t \wedge s_{m}}+M_{t \wedge s_{m}}
$$

with for $0 \leq t \leq T$

$$
\begin{array}{r}
A_{t}=\int_{0}^{t} \frac{\partial \rho_{n}^{(u)}}{\partial s}\left(s, Z_{s-}^{*}(u)\right) d s+\frac{1}{2} \int_{0}^{t} \frac{\partial^{2} \rho_{n}^{(u)}}{\partial x^{2}}\left(s, Z_{s-}^{*}(u)\right) d\left\langle Z^{*, c}(u)\right\rangle_{s}+ \\
\int_{0}^{t} \int_{\mathbb{R}}\left[\rho_{n}^{(u)}\left(s, Z_{s-}^{*}(u)+y\right)-\rho_{n}^{(u)}\left(s, Z_{s-}^{*}(u)\right)-\frac{\partial \rho_{n}^{(u)}}{\partial x}\left(s, Z_{s-}^{*}(u)\right) y\right] \nu^{Z^{*}, \mathbb{Q}_{u}^{*}}(d s, d y)
\end{array}
$$

and

$$
\begin{gathered}
M .=\int_{0}^{t} \frac{\partial \rho_{n}^{(u)}}{\partial x}\left(s, Z_{s-}^{*}(u)\right) d Z_{s-}^{*}(u)+ \\
\int_{0}^{t} \int_{\mathbb{R}}\left[\rho_{n}^{(u)}\left(s, Z_{s-}^{*}(u)+y\right)-\rho_{n}^{(u)}\left(s, Z_{s-}^{*}(u)\right)\left(\mu^{Z^{*}}(d s, d y)-\nu^{Z^{*}, \mathbb{Q}_{u}^{*}}(d s, d y)\right)\right.
\end{gathered}
$$

But since $A$ is predictable process and $\left(E_{\mathbb{Q}_{u}^{*}}\left(\phi_{n}\left(Z_{T}^{*}(u)\right) \mid \mathcal{G}_{t \wedge s_{m}}\right)\right)_{t \geq 0}$ is a $\mathbb{Q}_{u^{-}}^{*}$ martingale, we obtain that $\mathbb{Q}_{u}^{*}$-a.s., $A_{t}=0$ for all $0 \leq t \leq T$.

From [50], corollary 2.4 , p. 59, we get since $\sigma\left(\cup_{m=1}^{\infty} \mathcal{G}_{t \wedge s_{m}}\right)=\mathcal{G}_{t}$ that

$$
\lim _{m \rightarrow \infty} \rho_{n}^{(u)}\left(t \wedge s_{m}, Z_{t \wedge s_{m}}^{*}(u)\right)=E_{\mathbb{Q}^{*}}\left(\phi_{n}\left(Z_{T}^{*}(u)\right) \mid \mathcal{G}_{t}\right)
$$

Moreover, we remark that for all $x \in \mathbb{R}$ and $s \in[0, T]$

$$
\frac{\partial \rho_{n}^{(u)}}{\partial x}(s, x)=\xi_{s}^{(n, u)}(x)
$$

and all $x, y \in \mathbb{R}$ and $s \in[0, T]$

$$
H_{s}^{(n, u)}(x, y)=\rho_{n}^{(u)}\left(s, x Y_{s}^{*}(y)\right)-\rho_{n}^{(u)}(s, x)
$$

Using the definition of local martingales we conclude that the decomposition of Lemma holds. 
The next step consists to pass to the limit in previous decomposition. For that let us denote for $0 \leq t \leq T$

$$
\xi_{t}^{(u)}(x)=\mathbb{E}\left[\eta_{T-t}^{2}(u) f^{\prime \prime}\left(x \eta_{T-t}(u)\right)\right]
$$

and

$$
H_{t}^{(u)}(x, y)=\mathbb{E}\left(\eta_{T-t}(u)\left[f^{\prime}\left(x \eta_{T-t}(u) Y_{t}^{*}(y)\right)-f^{\prime}\left(x \eta_{T-t}(u)\right)\right]\right)
$$

Lemma 7. We have $\mathbb{Q}_{u}^{*}$-a.s., for all $t \leq T$,

$$
\begin{gathered}
E_{\mathbb{Q}_{u}^{*}}\left(f^{\prime}\left(Z_{T}^{*}(u)\right) \mid \mathcal{G}_{t}\right)=E_{\mathbb{Q}_{u}^{*}}\left[f^{\prime}\left(Z_{T}^{*}(u)\right)\right]+ \\
\int_{0}^{t} \beta_{s}^{*}(u) Z_{s-}^{*}(u) \xi_{s}^{(u)}\left(Z_{s-}(u)\right) d X_{s}^{(c), \mathbb{Q}_{u}^{*}}+\int_{0}^{t} \int_{\mathbb{R}} H_{s}^{(u)}\left(Z_{s-}(u), y\right)\left(\mu^{X}-\nu^{X, \mathbb{Q}_{u}^{*}}\right)(d s, d y)
\end{gathered}
$$

where $\nu^{X, \mathbb{Q}_{u}^{*}}$ is a compensator of the jump measure $\mu^{X}$ with respect to $\left(\mathbb{F}, \mathbb{Q}_{u}^{*}\right)$.

Proof. The proof consists to show the convergence in probability of stochastic integrals and conditional expectations using the properties of $\phi_{n}$ cited in Lemma 5 and can be performed in the same way as in [6].

\subsection{Optimal strategies in a change-point situation for initially enlarged filtration}

Let $u$ be a utility function belonging to $C^{3}(] \underline{x},+\infty[)$ and $f$ its convex conjugate, $f \in$ $C^{3}\left(\mathbb{R}^{+, *}\right)$. We suppose that $\mathcal{M}(P) \neq \emptyset$ and $\mathcal{M}(\tilde{P}) \neq \emptyset$ and we introduce the following hypotheses

$\left(\mathcal{H}_{4}\right)$ : For each compact set $K$ of $\mathbb{R}^{+, *}$ we have:

$$
\sup _{\lambda \in K} \sup _{t \in[0, T]} E_{Q^{*}}\left[\zeta_{t}^{*} f^{\prime \prime}\left(\lambda \zeta_{t}^{*}\right)\right]<\infty, \sup _{\lambda \in K} \sup _{t \in[0, T]} E_{\tilde{Q}^{*}}\left[\tilde{\zeta}_{t}^{*} f^{\prime \prime}\left(\lambda \tilde{\zeta}_{t}^{*}\right)\right]<\infty
$$

where $\zeta^{*}$ and $\tilde{\zeta} *$ are the densities of the $f$-divergence EMM's $Q^{*}$ and $\tilde{Q}^{*}$ with respect to $P$ and $\tilde{P}$ respectively.

Theorem 5. Let $u$ be a strictly concave function belonging to $C^{3}(] \underline{x},+\infty[)$. Suppose that a convex conjugate $f$ of $u$ satisfy $\left(\mathcal{H}_{1}\right),\left(\mathcal{H}_{2}\right),\left(\mathcal{H}_{3}\right),\left(\mathcal{H}_{4}\right)$ and (33). Then for change-point model (4) there exists an $\mathbb{F}$-optimal strategy $\phi^{*}$. If $c \neq 0$, then

$$
\phi_{t}^{*}=-\lambda \frac{\beta_{t}^{*}(\tau) Z_{t-}^{*}(\tau)}{S_{t-}} \xi_{t}^{(\tau)}\left(\lambda Z_{t-}^{*}(\tau)\right)
$$

with $\beta^{*}$ defined in Lemma 4 and $\lambda$ such that $\mathbb{E}_{\mathbb{Q}^{*}}\left(-f^{\prime}\left(\lambda Z_{T}^{*}(\tau)\right)\right)=x$.

If $c=0$ and $\operatorname{sipp}(\nu) \neq \emptyset$, supp $(\tilde{\nu}) \neq \emptyset$ both supports containing 0 , and $Y, \tilde{Y}$ are not identically 1 , then $f^{\prime \prime}(x)=a x^{\gamma}$ with $a>0$ and $\gamma \in \mathbb{R}$, and the optimal strategies are defined by the same formula but with the replacement of $\beta_{t}^{*}(\tau)$ by $\alpha_{t}^{*}(\tau)$ such that

$$
\alpha_{t}^{*}(\tau)=e^{-y_{0}} Y^{*}\left(y_{0}\right)^{\gamma} \frac{d Y^{*}}{d y}\left(y_{0}\right) \mathbf{1}_{\{\tau>t\}}+e^{-y_{1}} \tilde{Y}^{*}\left(y_{1}\right)^{\gamma} \frac{d \tilde{Y}^{*}}{d y}\left(y_{1}\right) \mathbf{1}_{\{\tau \leq t\}}
$$

with any $y_{0} \in \operatorname{supp}(\nu)$ and $y_{1} \in \operatorname{supp}(\tilde{\nu})$. 
Proof From Theorem 2 and the hypotheses $\left(\mathcal{H}_{1}\right),\left(\mathcal{H}_{2}\right),\left(\mathcal{H}_{3}\right)$ and (33) it follows that there exists an $f$-divergence minimal martingale measure $\mathbb{Q}^{*}$. Since the processes $X$ and $S$ are $\mathbb{F}$-adapted, applying Theorem 3.1 in [27], we have the existence of an $\mathbb{F}$-adapted optimal strategy $\phi^{*}$ such that

$$
-f^{\prime}\left(\lambda Z_{T}^{*}(\tau)\right)=x+\int_{0}^{T} \phi_{u}^{*} d S_{u}
$$

and such that $\int_{0} \phi_{u}^{*} d S_{u}$ defines a local martingale with respect to $(\mathbb{Q}, \mathbb{F})$. Then, for $c \neq 0$, we compare the decomposition of Lemma 7 and the decomposition of Theorem 3 to get our formulas. For $c=0$ we use first the Theorem 3 of [5] to prove that $f^{\prime \prime}(x)=$ $a x^{\gamma}$. Then, again we compare the decomposition of Lemma 7 and the decomposition of Theorem 3 to get our formulas.

Corollary 7. Let $u$ be common utility function and let $f$ be its convex conjugate, $f^{\prime \prime}(x)=a x^{\gamma}$, where $a>0$ and $\gamma \in \mathbb{R}$. Then for $x>\underline{x}$ there exists an u-asymptotically optimal strategy if and only if the conditions of Theorem 1 are verified for both processes $L$ and $\tilde{L}$. Furthermore, if $c \neq 0$ then

$$
\phi_{t}^{*}=-A_{t}(\tau) \frac{\beta_{t}^{*}(\tau)\left(Z_{t-}^{*}(\tau)\right)^{\gamma+1}}{S_{t-}}
$$

with

$$
A_{t}(\tau)=\alpha_{\gamma}(x) \frac{\mathbb{E}\left(\left[z_{T}^{*}(\tau) / z_{t}^{*}(\tau)\right]^{\gamma+2} \mid \tau\right)}{\mathbb{E}\left(\left[Z_{T}^{*}(\tau)\right]^{\gamma+2}\right)}
$$

If $c=0$, then we have the same formula for $\phi_{t}^{*}$ with replacement of $\beta_{t}^{*}(\tau)$ by $\alpha_{t}^{*}(\tau)$ given in Theorem [5. In addition, $\phi^{*}$ is optimal as soon as $\gamma \neq-1$.

Proof According to the Theorem 1, under the assumptions (43), (44) and (45), the Levy model associated with $L$ has an $f$-divergence minimal equivalent martingale measure which preserves the Levy property and is scale invariant. The same is true for the Levy model associated with $\tilde{L}$. Then, the existence of $f$-divergence EMM's for change-point model follows from Theorem 2 and the formulas for strategies follow directly from Theorem 5,

Remark 2. From Corollary 7 and Theorem 4 we can see the following. Let $\psi^{*}$ and $\tilde{\psi}^{*}$ be u-optimal strategies for the exponential Levy models $L$ and $\tilde{L}$ respectively. Then the u-optimal strategy for corresponding change-point model can be written as

$$
\phi_{t}^{*}=B_{t}(\tau) \psi_{t}^{*} \mathbf{1}_{\{\tau>t\}}+\tilde{B}_{t}(\tau) \tilde{\psi}_{t}^{*} \mathbf{1}_{\{\tau \leq t\}}
$$

where

$$
\begin{gathered}
B_{t}(\tau)=\left(c^{*}(\tau)\right)^{\gamma+1} \frac{\mathbb{E}\left(\left[\zeta_{\tau}^{*}\right]^{\gamma+2} \mid \tau\right) \mathbb{E}\left(\left[\tilde{\zeta}_{T-\tau}^{*}\right]^{\gamma+2} \mid \tau\right)}{\mathbb{E}\left(\left[Z_{T}^{*}(\tau)\right]^{\gamma+2}\right)} \\
\tilde{B}_{t}(\tau)=\left(c^{*}(\tau)\right)^{\gamma+1}\left(\frac{\zeta_{\tau}}{\tilde{\zeta}_{\tau}}\right)^{\gamma+1} \frac{\mathbb{E}\left(\left[\tilde{\zeta}_{T}^{*}\right]^{\gamma+2}\right)}{\mathbb{E}\left(\left[Z_{T}^{*}(\tau)\right]^{\gamma+2}\right)}
\end{gathered}
$$


When $u$ is exponential utility, and, hence, $\gamma=-1$, we see that $B_{t}(\tau)=\tilde{B_{t}} \tilde{(\tau)}=1$ and the optimal strategy $\phi^{*}$ can be obtained by pasting together two optimal strategies $\psi^{*}$ and $\tilde{\psi}^{*}$ at $\tau$. In addition, in this case $\phi^{*}$ is already adapted with respect to progressively enlarged filtration.

Example: Optimal strategy for Black-Scholes model with change point and exponential utility. As before, we now want to apply the results when $L$ and $\tilde{L}$ define Black-Scholes type models. Therefore, we assume that $L$ and $\tilde{L}$ are continuous Levy processes with characteristics $(b, c, 0)$ and $(\tilde{b}, c, 0)$ respectively. Let $\tau$ be a random variable bounded by $T$ which is independent from $L$ and $\tilde{L}$. Then the asymptotically optimal strategy from the point of view of maximization of exponential utility $u(x)=1-\exp (-x)$ will be :

$$
\phi_{t}^{*}=-\frac{\beta_{t}}{S_{t-}}=\frac{(b+c / 2) \mathbf{1}_{\llbracket 0, \tau \rrbracket}(t)+(\tilde{b}+c / 2) \mathbf{1}_{\rrbracket \tau,+\infty \llbracket}(t)}{c S_{t-}}
$$

\section{Acknowledgements}

The authors would like to thank Referees of this article for useful remarks and comments.

This work is supported in part by ECOS project M07M01 and by ANR-09-BLAN0084-01 of the Department of Mathematics of Angers's University.

\section{References}

[1] S. Ankirchner, C. Blanchet-Scalliet, A. Eyraud-Losel. Credit risk premium and quadratic BSDE's with a single jump. Working paper.

[2] M. Basseville, I. V. Nikiforov. Detection of abrupt changes - theory and applications. Prentice Hall, Englewood Cliffs, NJ, 1993.

[3] E. Bayraktar, S. Dayanik, I. Karatzas. The standard Poisson disorder problem revisited. Stoch. proc. Appl. 115, 9 (2005) 1437-1450.

[4] P. Carr, H. Geman, D. Madan, M. Yor. Stochastic volatility for Levy processes. Mathematical Finance, 13, 2 ( 2003) 345-382.

[5] S. Cawston, L. Vostrikova. Levy preservation and associated properties for $f$-divergence minimal equivalent martingale measures. Working paper.

[6] S. Cawston, L. Vostrikova. $f$-divergence approach and optimal portfolios in exponential Levy models. Working paper.

[7] A. Cerny, J. Kallsen. On the structure of general mean-variance hedging strategies. Cass Business School Research Paper. Available at SSRN: http://ssrn.com/abstract $=882762$. 
[8] J. Chen, A. Gupta. Statistical inference of covariance change points in Gaussian model. Statistics 38,1 (2004) 17-28.

[9] J. Chen, A. Gupta. Testing and Locating Variance changepoint with Application to Stock prices. Journal of the American Statistical Association 92 (1997)

[10] X. Chen, J. Wan. Option pricing for Time-change Exponential Levy Model under MEMM. Acta Mathematicae Applicatae Sinica, English Series, 23, 4 (2007) 651-664

[11] T. Choulli, C. Stricker. Minimal entropy-Hellinger martingale measure in incomplete markets. Mathematical Finance, 15 ( 2005) 465-490.

[12] T. Choulli, C. Stricker, J. Li. Minimal Hellinger martingale measures of order q. Finance Stoch. 11, 3 (2007) 399-427.

[13] CzIsar I. Information-type measure of divergence of probability distributions. MTA Oztaly Kezlemenyei 17 (1987) 123-149, 267-299.

[14] M. H. A. Davis. A note on the Poisson disorder problem. Banach Center Publ. 1 (1976) 65-72.

[15] A. Dias, P. Embrechts. Change-point analysis for dependence structures in finance and insurance. In: Risk Measures for the 21st Century, ed. by Giorgio Szegoe, Wiley Finance Series, 321-335, 2004.

[16] E. Eberlein. Jump-type Levy processes. In Handbook of Financial Series. Springer-Verlag, 2007.

[17] N. El Karoui, M. Jeanblanc, Ying Jiao. What happens after a default: The conditional density approach. Stochastic processes and their applications. 120 (2010) 1011-1032.

[18] F. Esche, M. Schweizer. Minimal entropy preserves the Lévy property : how and why. Stochastic processes and their applications, 115, 2 (2005) 299-327.

[19] H. Follmer, M. Schweizer. Hedging of Contingent Claims under Incomplete information. in: M.H. Davis and R.J. Elliott(eds),"Applied Stochastic Analysis", Stochastics Monographs, vol.5, Gordon and Breach, London/New York (1991) 389414.

[20] T. Fujiwara, Y. Miyahara. The Minimal Entropy Martingale Measures for Geometric Levy Processes. Finance and Stochastics. 7 (2003) 509-531.

[21] L. J. Galchuk, B. V. Rozovsky. The disorder problem for a Poisson process. Theory probab. Appl. 16 (1971) 729-734.

[22] A. Gandy, U. Jensen, C. Lutkebohmert. A Cox model with a change-point Applied to an Actuarial Problem. Braz. J. Probab. Stat. 19 (2005) 93 -109. 
[23] P. V. Gapeev, G. Pechkir. The Wiener disorder problem with finite horizon. Stoch. stoch. Rep.76, 1 (2004) 59-75.

[24] Gasbarra D., Valkeila E., Vostrikova L. Enlargement of filtration and additional information in pricing models: Bayesian approach. In Kabanov Yu., Liptser R., Stoyanov D. From Stochastic Calculus to Mathematical Finance, 257-285, Springer-Verlag, 2006.

[25] H. Geman, D. Madan, M. Yor. Time changes for Levy processes. Mathematical Finance, 11, 1 (2001) 79-96.

[26] H. Geman, D. Madan, M. Yor. Stochastic volatility, jumps and hidden time changes. Finance Sthoch. 6 (2002) 63-90.

[27] T. Goll, L. Ruschendorf. Minimax and minimal distance martingale measures and their relationship to portfolio optimization. Finance and Stochastics, Vol. V.4 (2001) 557-581.

[28] P. Grandits. On martingale measures for stochastic processes with independent increments. Theory Probab. Appl. vol.44, 1 (1999) 39-50.

[29] B. Grigelionis: Martingale characterisation of stochastic processes with independent increments. Litovsk. Math. Sb. 17 (1977) 75-86.

[30] X. Guo. A regime switching model: Statistical estimation, empirical evidence, and change point detection, Proc. SIAM-AMS-IMA Research Conference in Mathematical Finance, 139-155, 2004.

[31] D.M. Hawkins, K. D. Zamba. A change-Point Model for a Shift in Variance. Journal of Quality Technology, 37, 1 (2005) 21-31.

[32] F. Hubalek, C. Sgarra. Esscher transforms and the minimal entropy martingale measure for exponential Levy models Quantitative finance, 6, 2 (2006) $125-145$.

[33] J. Jacod, A. Shinyaev. Limit Theorems for Stochastic Processes. SpringerVerlag, 1987.

[34] M. Jeanblanc, S. Kloppel., Y. Miyahara. Minimal $F^{Q}$-martingale measures for exponential Levy processes. Ann. Appl. Probab. 17, 5/6 (2007), 1615-1638.

[35] J. Kallsen. Optimal portfolios for exponential Lévy processes. Mathematical Methods of Operations Research, 51 (2000) 357-374.

[36] J. Kallsen. Utility-based derivative pricing in incomplete markets. In H. Geman, D. Madan, S. Pliska, and T. Vorst, editors, Mathematical Finance - Bachelier Congress 2000, 313-338, Berlin, Springer 2002.

[37] J. Kallsen, A. Shiryaev. The cumulant process and Esscher's change of measure. Finance and Stochastics, 6 (2002) 397-428. 
[38] T. Kavtaradze , N. Lazrieva, M. Mania, P. Muliere. A Bayesianmartingale approach to the general disorder problem. Stochastic processes Appl. 117 (2007) ) , 1093-1120.

[39] R. Kitter Duality for Nonlinear Programming in a Banach Space. SIAM Journal on Applied Mathematics, 15, 2 ( 1967) 294-302.

[40] N. LazRieva, Thoronjadze. Optimal robust mean-variance hedging in incomplete financial markets. Journal of Mathematical Sciences 153, 3(2008) 262-290

[41] F. Liese, I. VAJdA. Convex statistical distances, Teubner Texte zur Mathematik 95. Teubner Verl. Leipzig ,1986.

[42] A. LokkA. Detection of disorder before an observable event. Preprint, $24 / 10 / 2006$.

[43] Y. Miyahara. Minimal Entropy Martingale Measures of Jump Type Price Process in Incomplete Assets Markets. Asian-Pacific Financial Markets. 6, 2 (1999) $97-113$.

[44] Y. Miyahara, A. Novikov. Geometric Levy Process Pricing Model. Proceedings of Steklov Mathematical Institute, 237 (2002) 176-191.

[45] E. S. PAGE. A test for a change in parameter occuring at an unknown point. Biometrika, 42 (1955) 523-527.

[46] E. S. PAGE. On problems in which a change in parameter occurs at an unknown point. Biometrika, 44 (1957) 248-252.

[47] G. Peskir, A. N. Shiryaev. Solving the Poisson disorder problem. Advance in finance and stochastics, Springer, Berlin, (2002) 295-312.

[48] M. Pollak, D. Siegmund. A diffusion process and its applications to detecting a change in the drift of Brownian motion. Bometrika, 72, 2 (1985) 267-280.

[49] S. W. RoBerTs. A comparison of some control chart procedures. Technometrics, 8 (1966) 411-430.

[50] D.Revuz, M.Yor. Continuous Martingales and Brownian Motion, SpringerVerlag, Berlin, 1999.

[51] K. Sato. Levy processes and infinitely divisible distributions CUP (1999).

[52] M. Schweizer. On the minimal martingale measure and the Follmer-Schweizer decomposition. Stochastic Analysis and Applications, 13 (1995), 573-599.

[53] M. Schweizer. A Guided Tour through Quadratic Hedging approaches, In: E. Jouini, J. Cvitanic, M. Musiela (eds.) :"Option Pricing, Interest Rates and Risk Management", Cambridge University Press, (1999) 538-574. 
[54] A. N. Shiryaev. On optimum methods in quickest detection problems. Theory Probab. Appl., 8 (1963) 22-46.

[55] A. Shiryaev. Essentials of Stochastic Finance. World Scientific, Singapore, 1999.

[56] A. N. Shiryaev. From "Disorder" to Nonlinear Filtering and Martingale theory. Mathematical events in the twentieth century, Springer, Berlin, 2006.

[57] A. N. ShIRYAEv. Quickest detection problems in the technical analysis of financial data. Mathematical Finance-Bachelier Congress, 2000 (Paris), 487-521, Springer Finance, Springer, Berlin.

[58] A. N. Shiryaev. On stochastic models and optimal methods in the problems of the quickest detection, Theory of probability and their applications, 53, 3 (2009) $385-401$.

[59] V. G. Spokoiny. On sequential detection of a small disorder. Frontiers in Pure and Applied Probab. 1, 238-255, H. Niemi et al. (Eds) 1993 TVP/VSP.

[60] S. Sukparungsee, A. Novikov. On EWMA procedure for detection of a change in observations via martingale approach. KMITL Sci. J. 6, 2 (2006) 373-380. 